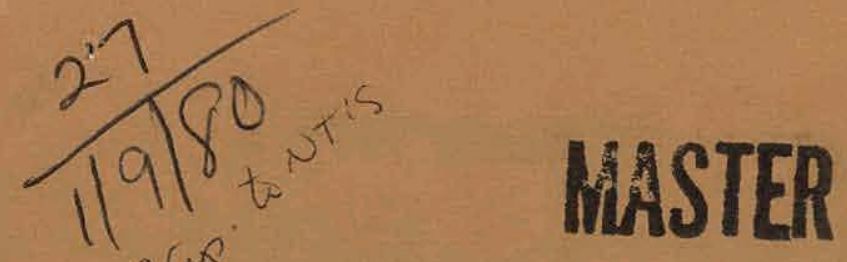

DOE/BG/01569-16

\title{
Western Gas Sands Project Quarterly Basin Activities Report
}

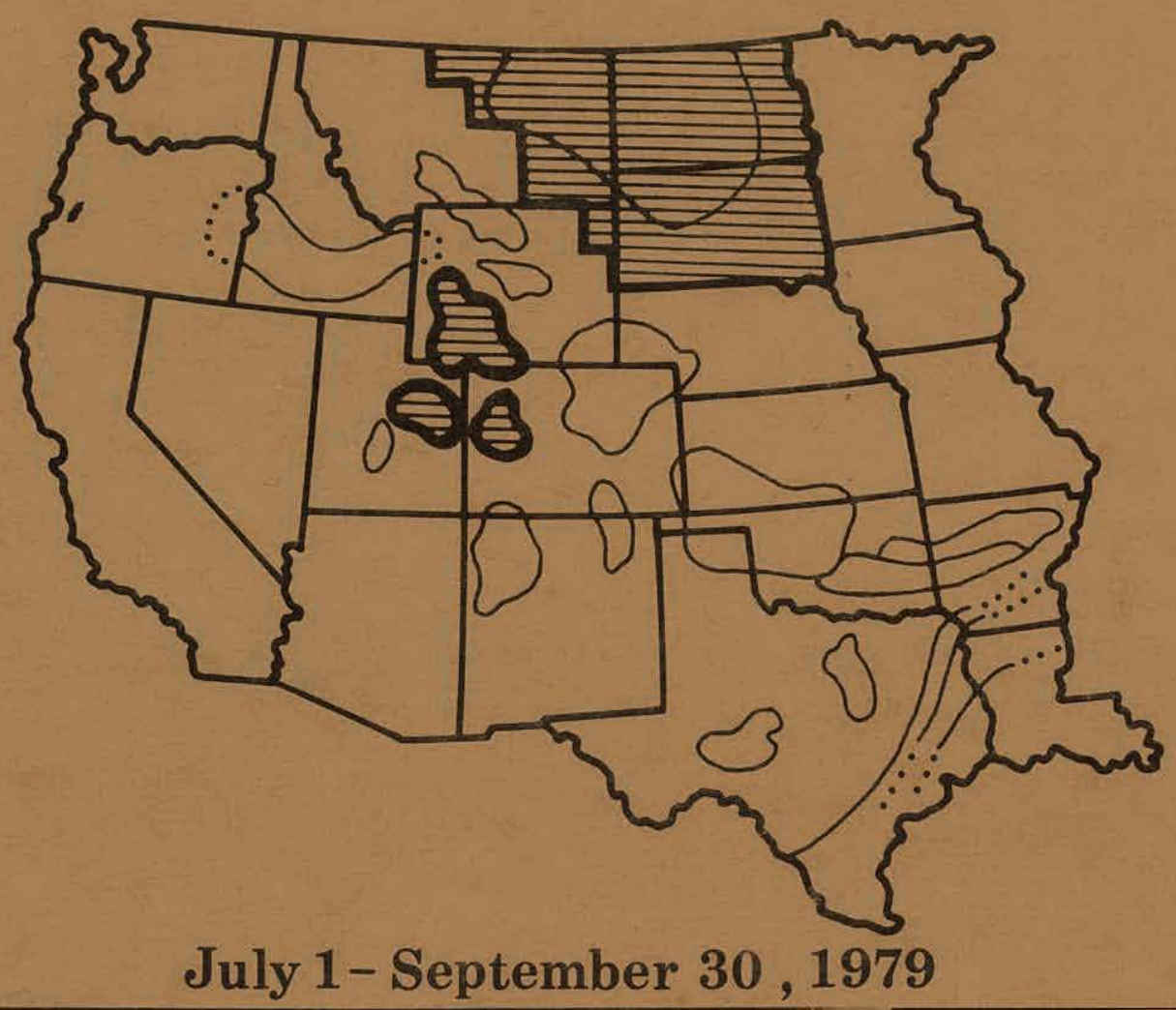

Prepared for

U.S. Department of Energy

Bartlesville Energy Technology Center

Charles II. Atkinsun

Project Manager

Compiled by CER Corporation

Las Vegas, Nevada

Contract DE-AC08-79BG01569

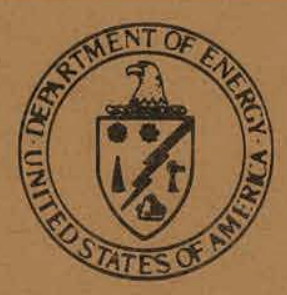




\section{DISCLAIMER}

This report was prepared as an account of work sponsored by an agency of the United States Government. Neither the United States Government nor any agency Thereof, nor any of their employees, makes any warranty, express or implied, or assumes any legal liability or responsibility for the accuracy, completeness, or usefulness of any information, apparatus, product, or process disclosed, or represents that its use would not infringe privately owned rights. Reference herein to any specific commercial product, process, or service by trade name, trademark, manufacturer, or otherwise does not necessarily constitute or imply its endorsement, recommendation, or favoring by the United States Government or any agency thereof. The views and opinions of authors expressed herein do not necessarily state or reflect those of the United States Government or any agency thereof. 


\section{DISCLAIMER}

Portions of this document may be illegible in electronic image products. Images are produced from the best available original document. 
This report was prepared as an account of work sponsored by the United States Government. Neither the United States nor the United States DOE, nor any of their employees, nor any of their contractors, subcontractors, or their employees, makes any warranty, express or implied, or assumes any legal liability or responsibility for the accuracy, completeness, or usefulness of any information, apparatus, product or process disclosed, or represents that its use would not infringe privately owned rights.

Available from the National Technical Information Service, U. S. Department of Commerce, Springfield, Virginia 22161.

NATIONAL TECHNICAL INFORMATION SERVICE PAPER COPY PRICES EFFECTIVE JANUARY 1,1979

$\begin{array}{cc}\begin{array}{c}\text { Page } \\ \text { Range }\end{array} & \begin{array}{c}\text { Domestic } \\ \text { Price }\end{array} \\ & \\ 001-025 & \$ 4.00 \\ 026-050 & \$ 4.50 \\ 051-075 & \$ 5.25 \\ 0,76-100 & \$ 6.00\end{array}$

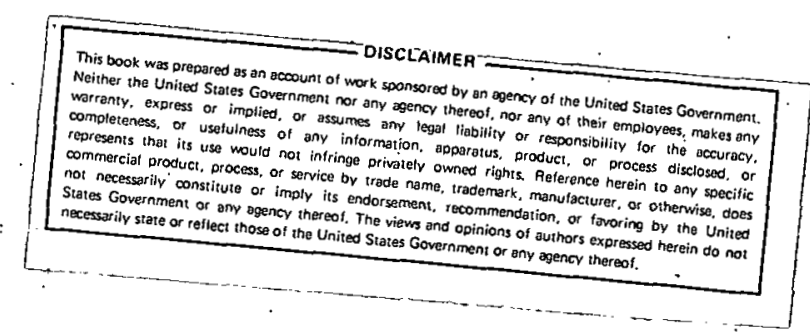




\section{Contents}

Page

1. INTRODUCTION $\ldots \ldots \ldots \ldots \ldots \ldots \ldots \ldots \ldots \ldots \ldots \ldots \ldots \ldots \ldots$

2. NORTHERN GREAT PLAINS PROVINCE $\ldots \ldots \ldots \ldots \ldots \ldots \ldots \ldots$

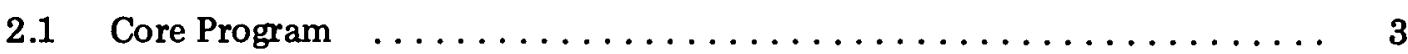

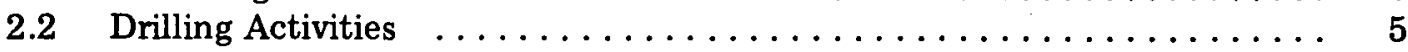

3. GREATER GREEN RIVER BASIN $\ldots \ldots \ldots \ldots \ldots \ldots \ldots \ldots \ldots \ldots \ldots$

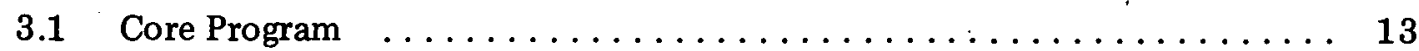

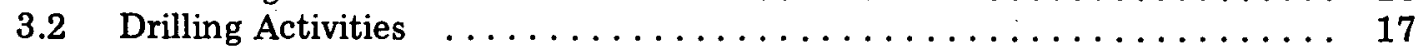

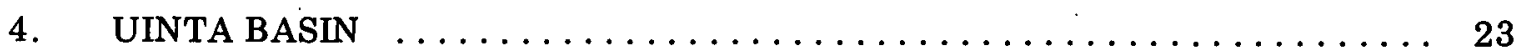

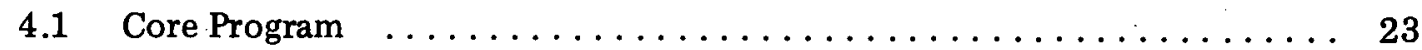

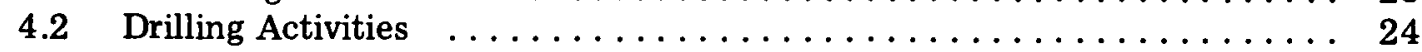

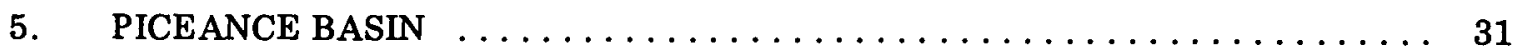

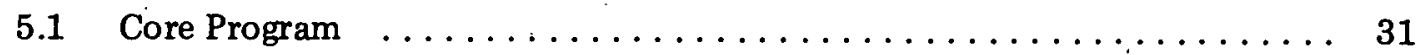

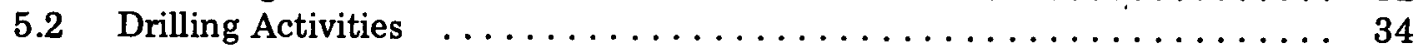




\section{Figures}

Page

Figure 1-1 Map of Western United States, Showing Areas of Interest $\ldots \ldots \ldots \ldots$.

Figure 2-1 USGS Designated Core Areas and Wells of Interest, Northern Great Plains Province $\ldots \ldots \ldots \ldots \ldots \ldots \ldots \ldots \ldots \ldots$

Figure 2-2 Correlation Chart of Cretaceous Rocks of the Northern Great

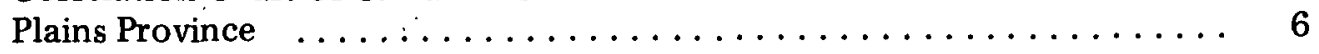

Figure 2-3 Wells Completed and Staked, USGS Designated Core Areas, Northern Great Plains Province $\ldots \ldots \ldots \ldots \ldots \ldots \ldots \ldots$

Figure 3-1. USGS Designated Core Areas and Wells of Interest, Greater Green River Basin . . . . . . . . . . . . . . . . . . . 14

Figure 3-2 Wells Completed and Staked, USGS Designated Core Areas, Greater Green River Basin $\ldots \ldots \ldots \ldots \ldots \ldots \ldots \ldots \ldots \ldots \ldots$

Figure 3-3 Stratigraphic Correlation Chart of Greater Green River,

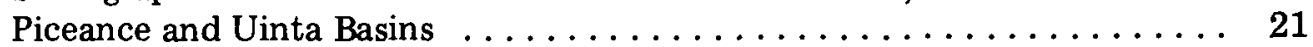

Figure 4-1 USGS Designated Core Areas and Wells of Interest, Uinta Basin $\ldots \ldots \ldots 25$

Figure 4-2 Wells Completed and Staked, USGS Designated Core Areas, Uinta Basin . ......................... 29

Figure 5-1 USGS Designated Core Areas and Wells of Interest,

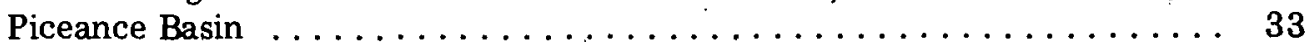

Figure 5-2 Wells Completed and Staked, USGS Designated Core Areas,

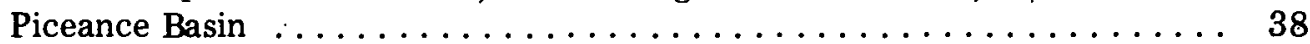




\section{Tables}

Page

Table 2-1 Summary of Wells Located in USGS Designated Core Areas-

Northern Great Plains Province $\ldots \ldots \ldots \ldots \ldots \ldots \ldots \ldots \ldots$

Table 2-2 Summary of Drilling Activities, Northern Great Plains Province $\ldots \ldots \ldots 7$

Table 2-3 Summary of Wells Completed-Northern Great Plains Province $\ldots . \ldots .9$

Table 2-4 Summary of Active Wells and New Locations-Northern Great

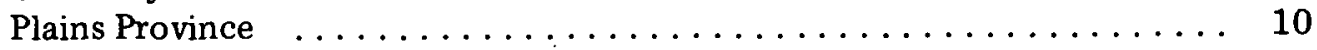

Table 3-1 Summary of Wells Located in USGS Designated Core Areas-

Greater Green River Basin $\ldots \ldots \ldots \ldots \ldots \ldots \ldots \ldots$

Table 3-2 Summary of Drilling Activities, Greater Green River Basin $\ldots \ldots \ldots \ldots$

Table 3-3 Summary of Wells Completed-Greater Green River Basin $\ldots \ldots \ldots \ldots 17$

Table 3-4 Summary of Active Wells and New Locations-Greater Green

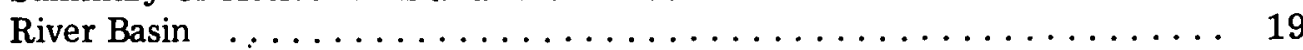

Table 4-1 Summary of Wells Located in USGS Designated Core Areas-

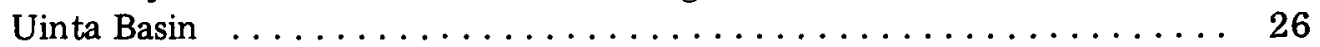

Table 4-2 Summary of Drilling Activities, Uinta Basin $\ldots \ldots \ldots \ldots \ldots \ldots \ldots$

Table 4-3 Summary of Wells Completed-Uinta Basin $\ldots \ldots \ldots \ldots \ldots \ldots \ldots$

Table 4-4 Summary of Active Wells and New Locations-Uinta Basin . . . . . . . 28

Table 5-1 Summary of Wells Located in USGS Designated Core Areas-

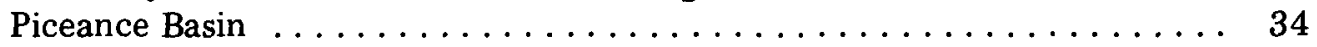

Table 5-2 Summary of Drilling Activities, Piceance Basin $\ldots \ldots \ldots \ldots \ldots \ldots \ldots$

Table 5-3 Summary of Wells Completed-Piceance Basin $\ldots \ldots \ldots \ldots \ldots \ldots$

Table 5-4 Summary of Active Wells and New Locations-Piceance Basin . . . . . 37 


\section{Abbreviations and Acronyms}

\section{Completed Wells}

$\begin{array}{ll}\text { WF } & \text { unsuccessful new field wildcat } \\ \text { WFD } & \text { new field discovery } \\ \text { WD } & \text { unsuccessful deeper pool wildcat } \\ \text { WDD } & \text { deeper pool discovery } \\ \text { WS } & \text { unsuccessful shallower pool wildcat } \\ \text { WSD } & \text { shallow pool discovery } \\ \text { WP } & \text { unsuccessful new pool wildcat } \\ \text { WPD } & \text { new pool discovery } \\ \text { WO } & \text { unsuccessful wildcat outpost } \\ \text { WOE } & \text { wildcat outpost extension } \\ \text { D } & \text { unsuccessful development well } \\ \text { DG } & \text { development gas well }\end{array}$

\section{Drilling/Staked Wells}

$\begin{array}{ll}\text { WF } & \text { new field wildcat } \\ \text { WD } & \text { deeper pool wildcat } \\ \text { WS } & \text { shallower pool wildcat } \\ \text { WP } & \text { new pool wildcat } \\ \text { WO } & \text { wildcat outpost } \\ \text { D } & \text { development }\end{array}$

BHC Borehole Compensated Log

$\mathrm{Cp} \quad$ Drilling completed

DIL Dual Induction Laterlog

D\&A Dry and Abandoned

FDC Formation Density Log

Fr First Report

GR Gamma Ray

PB Plugged back

SDR Shut down for repairs

SDW Shut down for weather

SI Shut in

Spud Commencement of drilling

Sp Spud In

SP Spontaneous Potential

SN Sidewall Neutron

Swb(d)(g) Swabbed, swabbing

TD Total Depth

Tstg Testing

WOCT Waiting on completion tools

WOO Waiting on orders 


\section{Introduction}

This quarterly basin activities report is a summation of three months drilling and testing activities in the four primary study areas of the WGSP. This report details information in each study area for the months of July, August, and September, 1979. Refer to Figure 1-1 for the location of study areas in the WGSP.

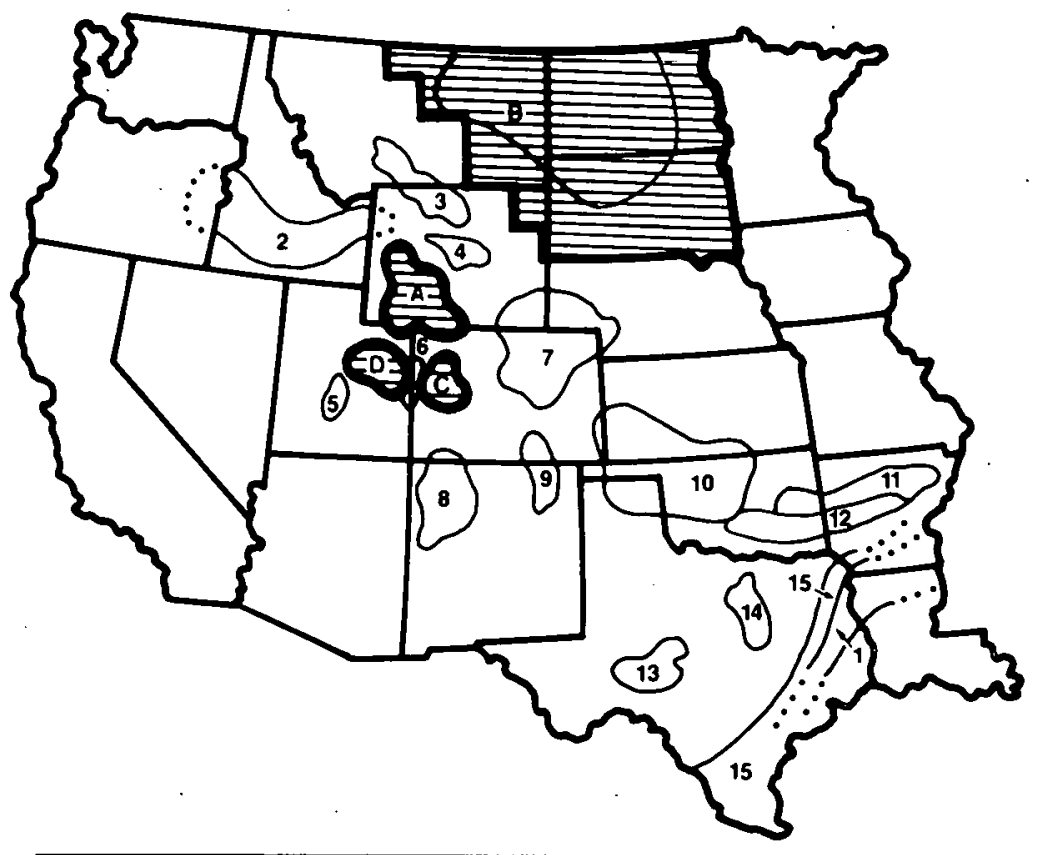

PRIMARY STUDY AREAS

A. Greater Green River Basin

B. Northern Great Plains Province

C. Piceance Basin

D. Uinta Basin

ADDITIONAL LOW PERMEABILITY SANDSTONE AREAS

1. Cotton Valley Trend

2. Snake River Downwarp

3. Big Hom Basin

4. Wind River Basin

5. Wasatch Plateau

6. Douglas Creek Arch

7. Denver Basin

8. San Juan Basin

9. Raton Basin

10. Anadarko Basin

11. Arkoma Basin

12. Ouachita Mountains Province

13. Sonora Basin

14. Fort Worth Basin

15. Western Gulf Basin

Figure 1-1 Map of Western United States, Showing Areas of Interest

The monitoring of basin activities is an important part of resource assessment and promotes cooperation between industry and government. This is especially evident in the core program. Certain areas in each study area have been recommended by the USGS for obtaining core. Operators active in these specific areas are located by monitoring new activity in each area and contacted by CER Corporation to negotiate contracts for a joint industry/ government coring operation. 
The summary of information on each study area is divided into two sections. The core program section identifies industry activity within the preferred core areas and relates the status of WGSP core acquisition developments. The second section details drilling and testing operations of interest and tabulates production figures from horizons of interest. Newly staked and completed wells are listed and located on a map with red and blue numbers, respectively.

The drilling information used in this report was compiled primarily from The Rocky Mountain Region Report, published daily by Petroleum Information Corporation. Additional sources used were the Montana Oil and Gas Journal, The Oil and Gas Journal and the Western Oil Reporter.

Background and geologic information can be obtained from the Quarterly Basin Activities Reports, dated January 1, 1978 (NVO/0655-03), April 1, 1978, (NVO/0655-05) and July 21, 1978, (NVO/0655-06). Included in these reports are stratigraphic correlation charts, cross sections and a brief account of the geology of each area and information relating to the USGS recommended coring locations. For information on the core program, refer to the WGSP Core Program, January 12, 1978. These reports may be obtained from the Technical Information Center (TIC), Post Office Box 62, Oak Ridge, Tennessee, 37830, except the Core Program document, which is available from CER Corporation, 4220 South Maryland Parkway, Suite 801, Las Vegas, Nevada, 89109. 


\section{Northern Great Plains Province}

\subsection{CORE PROGRAM}

Specific areas within Montana were selected by the USGS for the initiation of the WGSP core program. These areas include:

- Powder River County - T5S, R52E, to T53E.

- Custer and Prairie Counties - T10N to T11N, R48E to R49E.

- Valley County - T29N to T30N, R35E to R37E.

- Phillips County - T29N to T30N, R26E to R27E.

The four areas (see Figure 2-1 for location) were chosen for the following reasons:

- They are removed from productive fields and little data is available in the areas.

- There are two or more potential reservoir intervals and adjacent source beds in the areas.

- The combination of these areas will test several different reservoir types, including sections with fine-grained carbonates, such as chalk, and fractured reservoirs found along lineament zones.

The objectives are Cretaceous horizons ranging from the Judith River Formation through the older Mowry Formation. Figure 2-2 is a correlation chart of Cretaceous rocks in the Northern Great Plains Province. Drilling depths to reach these objectives are shallower in the Province $(2,000-4,000 \mathrm{ft})$ than in the basins of Wyoming, Colorado and Utah. Therefore, costs for recovering core and obtaining complete log data should be less than in basins where the objectives are deeper. 


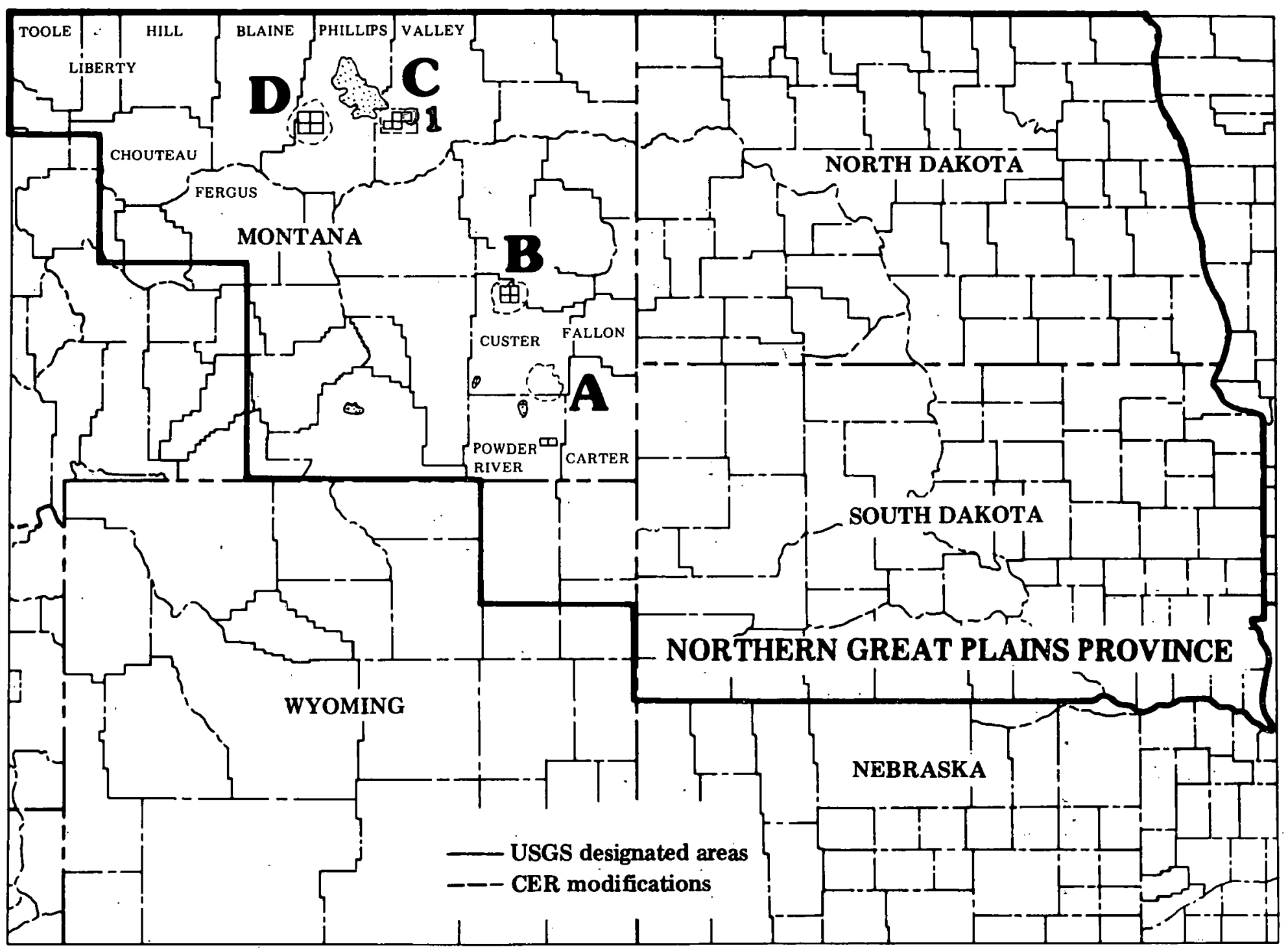

Figure 2-1 USGS Designated Core Areas and Wells of Interest, Northern Great Plains Province (refer to Table 2-1) 
In the Northern Great Plains Province, Chevron declined a coring contract for a well in southeast Montana, but will send any core that is taken to the USGS when their own studies have been completed. Midlands (Kansas-Nebraska) agreed to coring their Phillips County, Montana, well and a stratigraphic study of the area is in progress. B. Pickard, representing Sugar Creek Resources, contacted CER Corporation with a proposed Montana drilling site in response to the WGSP core program publicity in the Oil and Gas Journal.

Table 2-1 is a summary of wells located in USGS designated core areas and Figure 2-1 shows the location of these wells.

Table 2-1 Summary of Wells Located in USGS Designated Core Areas Northern Great Plains Province

\begin{tabular}{|c|c|c|c|c|c|c|c|c|c|}
\hline DATE & OPERATOR & WELL NAME & $\begin{array}{c}\text { MAP } \\
\text { INDEX } \\
\text { NO. }\end{array}$ & $\begin{array}{l}\text { LOCATION } \\
\text { Sec/T/R }\end{array}$ & $\begin{array}{l}\text { HORIZON } \\
\text { rojected Depth } \\
\text { coducing Interval) }\end{array}$ & $\begin{array}{c}\text { FINAL } \\
\text { TD }\end{array}$ & $\begin{array}{l}\text { FRACTURE } \\
\text { TREATMENT }\end{array}$ & COMMENTS & $\begin{array}{l}\text { IPF in } \\
\text { MCFD }\end{array}$ \\
\hline $\begin{array}{l}\text { FR: } \\
\text { 10-5-78 } \\
\text { SP: } \\
11-16-78\end{array}$ & $\begin{array}{l}\text { J. J. C. Paine } \\
\text { \& Associates }\end{array}$ & $\begin{array}{l}1-0407 \\
\text { Strommen }\end{array}$ & 1 & $\begin{array}{l}\text { nesw 4/30N/37E } \\
\text { Unnamed Field } \\
\text { Valley Cty. MT }\end{array}$ & $\begin{array}{l}\text { Greenhorn } \\
(1,375)\end{array}$ & 1,425 & . & $\begin{array}{l}\text { Wo. . WOCT, } \\
\text { located in core } \\
\text { area C. }\end{array}$ & \\
\hline $\begin{array}{l}\text { FR: } \\
10-5-78 \\
\text { SP: } \\
11-13-78\end{array}$ & $\begin{array}{l}\text { J. J. C. Paine } \\
\text { \& Associates }\end{array}$ & $\begin{array}{l}1-0507 \\
\text { Strommen }\end{array}$ & 1 & $\begin{array}{l}\text { sese } 5 / 30 N / 375 \\
\text { Unnamed Field } \\
\text { Valley Cty, MT }\end{array}$ & $\begin{array}{l}\text { Greenhorn } \\
(1,375)\end{array}$ & 1,425 & & $\begin{array}{l}\text { Wo. . woct. Wor. } \\
\text { located in core } \\
\text { area C. }\end{array}$ & \\
\hline
\end{tabular}

\subsection{DRILLING ACTIVITIES}

Production in the third quarter decreased 26 percent from the previous quarter. Although production from the Eagle and Blackleaf Formations increased substantially from last quarter, production from the Shannon and Phillips Formations decreased. The Eagle Formation contributed 56 percent of total production, Bow Island/Muddy contributed 34 percent of production, Blackleaf produced 7 percent, Carlile Formation produced 2 percent and Phillips contributed 1 percent towards total production.

Although the total production was down, the total of 75 completed wells only decreased by one from last quarter. Fifty-five were development wells, with 33 producing and 22 D\&A. Only 4 wildcat wells were producing by quarter's end, compared to the 16 wildcats declared D\&A. A summary of drilling activities for the Northern Great Plains Province is given in Table 2-2. 


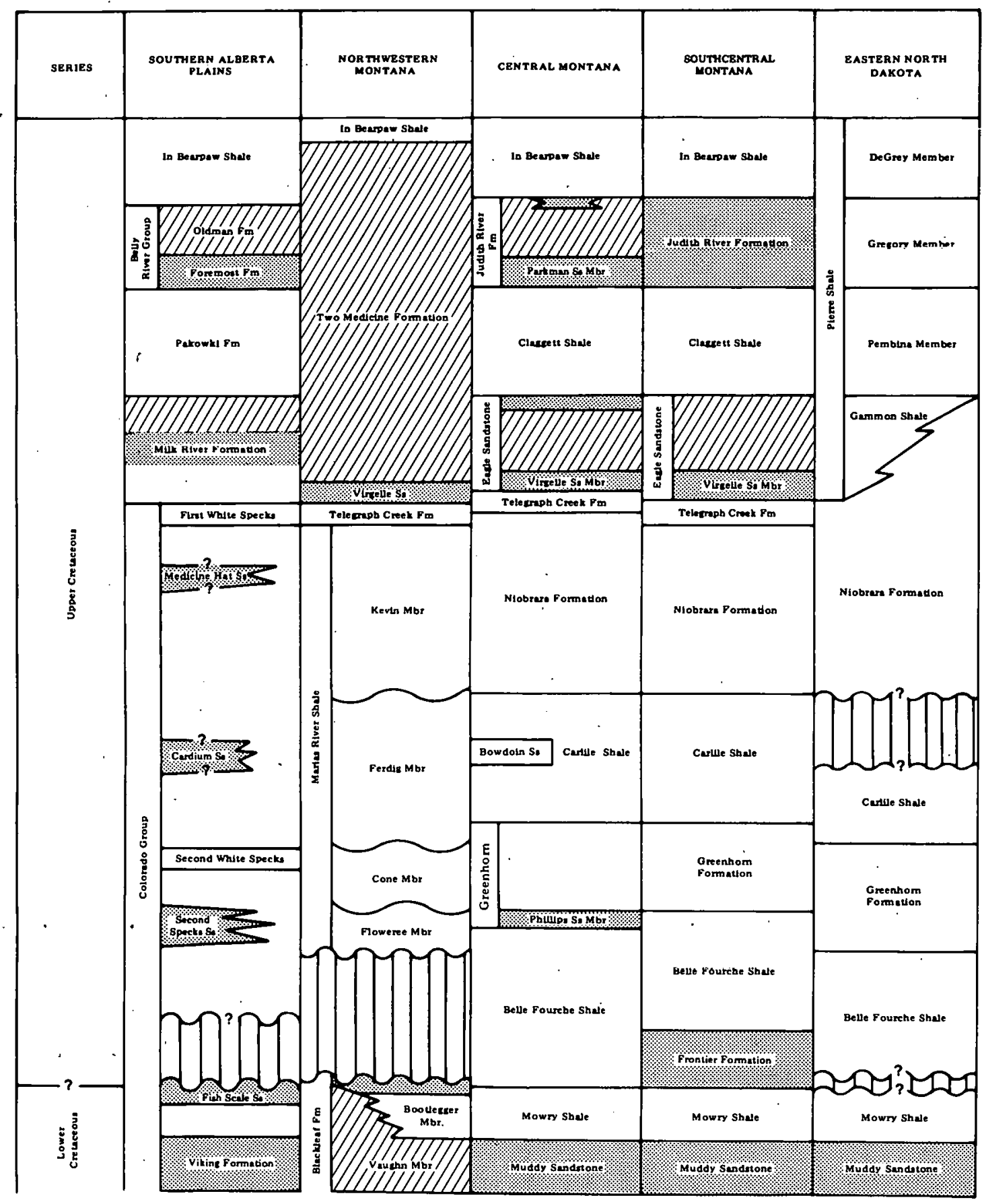

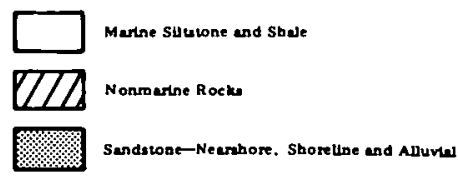

U.S.G.S. Investigation Chart OC-70, Published by the U.S. Geologic Survey, 1976.

Figure 2-2 Correlation Chart of Cretaceous Rocks of the Northern Great Plains Province 


\section{Table 2-2 Summary of Drilling Activities, Northern Great Plains Province *}

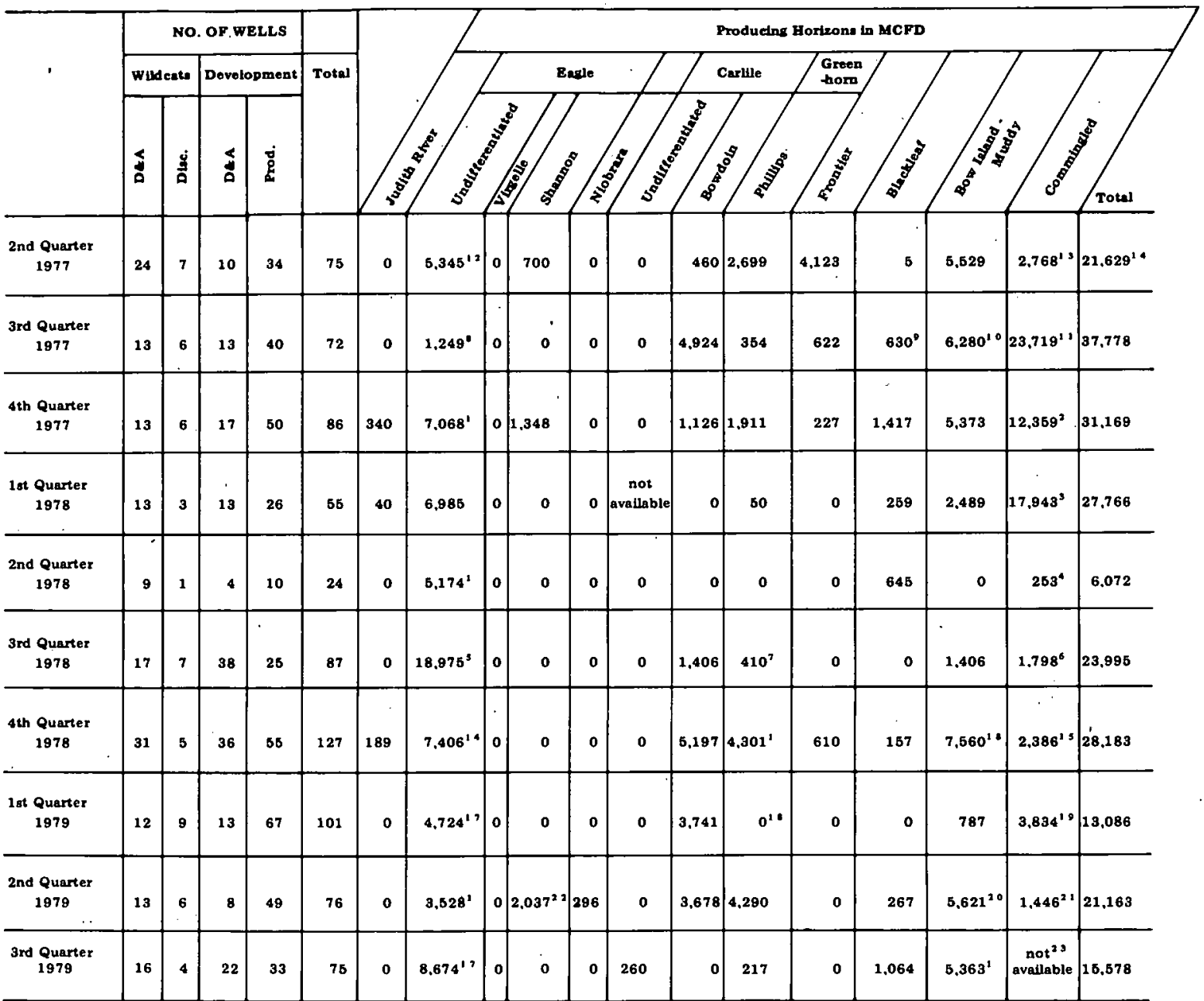

1. IPF not avallable trom one well.

2. Bowdoln/Greenhom

3. Judith River/Eagle - 60 Bowdoin/Phillips - 15,894 Bowdoin/Greenhorn/Phullipg - 844 Greenhom/Phillips - 1,145

4. Fish Scales/Bow laland

5. Natural production: 13,93

6. Bowdoin/Phillips - 284 Bowdoin/Greenhom - 368 Blackleat/Spike (natural) - 69 Bowdoin/Greenhom/Phulps - 44

7. 158 natural "White Specks"

8. Natural production
9. Natural production

10. $4,245 \cdot$ natural

11. Bowdoln/Greenhom/Phillps $-14,878$ Bowdoln/Greenhom - 1.180 Coloredo/Blactleat/Bows - 3.690 Colorado/2nd Bow Island - 436

12. Natural production $-4,560$

13. Bowdoln/Phillips - 205 Blackle at/Bow Island - 2.500 Judith River/Eazle - 63

14. Natural Production $-2,490$

16. Bowdoin/Phillips - 1,710 Bowdoln/Greenhom/Phillps - 676 Blackleaf/Bow Island - not availabte
16. IP $\bar{F}$ not avallable trom two well

17. IPF not available trom six wells

18. IPF not avallable from eleven wells

19. Bowdoin /Phillips - 960 Bowdoin/Greenhom - 711 Greenhom/Phllips - 2,078 Equle/Bowdoln - 85

20. Natural Produetion - 449

21. Bowdoin/Greenhom/Phillips - 1,361 Bowdoln/Eágle - 85

22. IPF not available trom five wello

23. Blackhead/Bow Island

- Dats compiled trom P. I. Rocky Mountains Region Report "Completions" 
Colorado Interstate Gas Exploration and Chevron Oil are continuing their exploration programs for Niobrara and Morrison objectives, respectively. Both are currently active in Custer and Rosebud Counties with CIGE also active in Garfield, Fallon and Carter Counties. Activity on the Bearpaw Arch and Bowdoin Dome area continues at a rate similar to previous quarters of 1979. Sugar Creek Resources has 3 Eagle tests $(2,000 \mathrm{ft})$ located on the west flank of Cedar Creek Anticline, Fallon County. These tests represent a new attempt to expand the Eagle, and possibly Judith River, production off the anticline. The original discovery well was completed in 1912 .

Joseph J. C. Paine has about 30 wells staked or in various stages of completion in the area of Little Missouri Field of Bowman County, North Dakota. The Eagle formation is the objective zone. In South Dakota, Jerry McCutchin, Jr., is continuing Shannon (lower Eagle) exploration in the West Short Pine Hills Field of Harding County, with 1 test currently planned to the deeper Greenhom formation. This test is located in Cady Creek Field, southeast of West Short Pine Hills Field.

The largest fracture treatment reported this quarter was performed on Joseph J. C. Paine \& Associates' No. 1 Chapman, Little Missouri Field, Bowman County, North Dakota. The well produced 368 MCFD of new gas after being fractured with 24,612 gal fluid and 75,000 lb sand.

Summaries of completed wells and active and newly located wells are listed in Tables $2-3$ and 2-4, respectively. Figure 2-3 shows the locations of these wells: 
Table 2-3 Summary of Wells Completed-Northern Great Plains Province

\begin{tabular}{|c|c|c|c|c|c|c|c|c|c|}
\hline DATE & OPERATOR & WELL NAME & $\begin{array}{c}\text { MAP } \\
\text { INDEX } \\
\text { NO. }\end{array}$ & $\begin{array}{l}\text { LOCATION } \\
\text { Sec/T/R } \\
\text { Ox Pro }\end{array}$ & $\begin{array}{l}\text { HORIZON } \\
\text { rolected Depth. } \\
\text { odueing Interval) }\end{array}$ & $\begin{array}{c}\text { FINAL } \\
\text { TD }\end{array}$ & $\begin{array}{l}\text { FRACTURE } \\
\text { TREATMENT }\end{array}$ & COMMENTS & $\begin{array}{l}\text { IPF in } \\
\text { MCFD }\end{array}$ \\
\hline $\begin{array}{l}\text { SP: } \\
7-15-78 \\
\text { CP: } \\
7-18-78\end{array}$ & $\begin{array}{c}\text { Cabot Corp. } \\
.\end{array}$ & $\begin{array}{l}\text { 1-22 Prince } \\
\text { and Sons }\end{array}$ & 1 & $\begin{array}{l}\text { nesw } 22 / 5 N / 27 E \\
\text { Wudcat Field } \\
\text { Stenley Cnty, sn }\end{array}$ & $\begin{array}{l}\text { Niobraxa } \\
(2,000)\end{array}$ & $(1,200)$ & . & $\begin{array}{l}\text { WF, D\&A, } \\
\text { Operator } \\
\text { completed } \\
2 \text { other } \\
2,000 \mathrm{tt} \\
\text { wells in this } \\
\text { locale, all } \\
\text { D\&A. }\end{array}$ & \\
\hline $\begin{array}{l}\text { SP: } \\
11-17-78 \\
\text { CP: } \\
11-21-78\end{array}$ & $\begin{array}{l}\text { Charles G. } \\
\text { Knight }\end{array}$ & 1 IRA. & 2 & $\begin{array}{l}\text { segw } 26 / 7 \mathrm{~S} / 52 \mathrm{E} \\
\text { Wildcat Field } \\
\text { Powder River Cnty, } \\
\text { MT }\end{array}$ & $\begin{array}{l}\text { Muddy } \\
(5,000)\end{array}$ & 5,050 & & WF, D\&A. & \\
\hline $\begin{array}{l}\text { SP: } \\
6-20-79 \\
\text { CP: } \\
6-22-79\end{array}$ & Devon Corp. & 1 Mees & 3 & $\begin{array}{l}\text { nwse } 1 / 22 N / 19 E \\
\text { Wildcat Field } \\
\text { Fergus Cnty, MT }\end{array}$ & $\begin{array}{l}\text { Eagle } \\
(2,000)\end{array}$ & 1,718 & . & $\begin{array}{l}\text { WF. D\&A. } \\
\text { no cores or } \\
\text { tests. }\end{array}$ & \\
\hline $\begin{array}{l}\text { SP: } \\
6: 17-79 \\
\text { CP: } \\
6-19-79\end{array}$ & Devon Corp. & 2 Glady Mees & 3 & $\begin{array}{l}\text { nwne } 2 / 22 \mathrm{~N} / 19 \mathrm{E} \\
\text { Widcat Field } \\
\text { Fergus Cnty. MT }\end{array}$ & $\begin{array}{l}\text { Eagle } \\
(2,000)\end{array}$ & 1.940 & . & $\begin{array}{l}\text { wo, no cores } \\
\text { or tests. }\end{array}$ & \\
\hline $\begin{array}{l}\text { SP: } \\
6-11-79 \\
\text { CP: } \\
6-13-79\end{array}$ & Devon Corp. & $\begin{array}{l}1 \text { c Olson } \\
\text { et al }\end{array}$ & 4 & $\begin{array}{l}\text { swse } 26 / 23 N / 19 E \\
\text { Leroy Field } \\
\text { Fergus Cnty, MT }\end{array}$ & $\begin{array}{l}\text { Eagle } \\
(2,000)\end{array}$ & 1,5654 & & Wo, D\&A. & \\
\hline $\begin{array}{l}\text { SP: } \\
\text { 6-26-79 } \\
\text { CP: } \\
6-28-79\end{array}$ & Devon Corp. & 1 Osburnsen & 5 & $\begin{array}{l}\text { swsw } 31 / 23 N / 20 E \\
\text { Widcat Field. } \\
\text { Fergus Cnty, MT }\end{array}$ & $\begin{array}{l}\text { Eagle } \\
(2,000)\end{array}$ & 1,950 & & $\begin{array}{l}\text { WF, D\&A. } \\
\text { no cores or } \\
\text { tests. }\end{array}$ & \\
\hline $\begin{array}{l}\text { SP: } \\
7-7-79 \\
\text { CP: } \\
7-8-79\end{array}$ & Balcron Oil & 2-5 Tweet & 6 & $\begin{array}{l}\text { sese } 6 / 29 \mathrm{~N} / 1 \mathrm{~W} \\
\text { Ledger Field } \\
\text { Pondera Cnty, MT }\end{array}$ & $\begin{array}{l}\text { Bow Lsland } \\
\text { (593-603) } \\
\text { open hole }\end{array}$ & 603 & & $\begin{array}{l}\text { DG, no logs } \\
\text { run, no cores } \\
\text { or tests, oper- } \\
\text { ator has } 16 \\
\text { additional } \\
\text { Bow Island } \\
\text { completions } \\
\text { in the area. }\end{array}$ & 130 \\
\hline $\begin{array}{l}\text { SP: } \\
6-16-79 \\
\text { CP: } \\
7-17-79\end{array}$ & $\begin{array}{l}\text { Tricentrol } \\
\text { U.S. }\end{array}$ & $\begin{array}{l}16-16 \\
\text { State }\end{array}$ & 7 & $\begin{array}{l}\text { sese } 16 / 30 N / 1 \text { 5E } \\
\text { Tiger Ridge } \\
\text { Hill Cnty, MT }\end{array}$ & $\begin{array}{l}\text { Eagle } \\
(1.442- \\
1,460)\end{array}$ & 1,800 & $\begin{array}{l}30,000 \mathrm{lb} \\
\text { sand }\end{array}$ & $\begin{array}{l}\text { DG, no cores } \\
\text { or tests, oper- } \\
\text { ator has } 5 \\
\text { other Eagle } \\
\text { completions } \\
\text { in the area. }\end{array}$ & 1.200 \\
\hline $\begin{array}{l}\text { SP: } \\
6-18-79 \\
\text { CP: } \\
6-23-79\end{array}$ & $\begin{array}{l}\text { J. Bums } \\
\text { Brown }\end{array}$ & $\begin{array}{l}\text { 19-32-19B } \\
\text { Mueller }\end{array}$ & 8 & $\begin{array}{l}\text { swnw } 19 / 32 \mathrm{~N} / 19 \mathrm{E} \\
\text { Black Coulee } \\
\text { Blaine Cnty, MT } \\
\end{array}$ & $\begin{array}{l}\text { Eagle } \\
(1,500)\end{array}$ & 1,390 & & $\begin{array}{l}\text { DG, D\&A, } \\
\text { no cores or } \\
\text { tests, oper- } \\
\text { ator has } 6 \\
\text { other Eagle } \\
\text { completions } \\
\text { in the area. }\end{array}$ & \\
\hline $\begin{array}{l}\text { SP: } \\
6-7-79 \\
\text { CP: } \\
8-22-79\end{array}$ & $\begin{array}{l}\text { Falcon- } \\
\text { Colorado } \\
\text { Exploration }\end{array}$ & 2-5 Bowman & 9 & $\begin{array}{l}\text { senw } 5 / 32 N / 35 E \\
\text { Swanson Creek } \\
\text { Field } \\
\text { Phillips Cnty, MT }\end{array}$ & $\begin{array}{l}\text { Phillips } \\
(1,225- \\
1,236)\end{array}$ & 1,430 & $\begin{array}{l}260 \mathrm{gal} \mathrm{acid} \text {, } \\
13,000 \mathrm{gal} \text { water. } \\
40,000 \mathrm{lb} \text { sand }\end{array}$ & $\begin{array}{l}\text { DG, no cores } \\
\text { or tests. }\end{array}$ & 233 \\
\hline $\begin{array}{l}\text { FR: } \\
6-9-78\end{array}$ & $\begin{array}{l}\text { J. Burns } \\
\text { Brown }\end{array}$ & $\begin{array}{l}3-32-15 \\
\text { State }\end{array}$ & 10 & $\begin{array}{l}\text { swne } 3 / 32 N / 16 E \\
\text { Unnamed Field } \\
\text { Hill Cnty, MT }\end{array}$ & $\begin{array}{l}\text { Eagle } \\
(1.600)\end{array}$ & & & $\begin{array}{l}\text { D, Aban- } \\
\text { doned loca- } \\
\text { tion. }\end{array}$ & \\
\hline $\begin{array}{l}\text { SP: } \\
6-12-79 \\
\text { CP: } \\
6-17-79\end{array}$ & $\begin{array}{l}\text { Grace } \\
\text { Petroleum }\end{array}$ & $\begin{array}{l}\text { 6-9 Van } \\
\text { Dressel }\end{array}$ & 11 & $\begin{array}{l}\operatorname{sen} w 9 / 34 N / 7 E \\
\text { Unnamed Field } \\
\text { Liberty Cnty, MT }\end{array}$ & $\begin{array}{l}\text { Bow Island } \\
(2,140)\end{array}$ & $\begin{array}{l}2,149 \\
\text {. }\end{array}$ & & D, D\&A. & \\
\hline $\begin{array}{l}\text { SP: } \\
12-19-78 \\
\text { CP: } \\
6-21-79\end{array}$ & $\begin{array}{l}\text { Odessa Natural } \\
\text { Corp. }\end{array}$ & 1 Ivanovitch & 12 & $\begin{array}{l}\text { nesw } 14 / 36 \mathrm{~N} / 32 \mathrm{E} \\
\text { Wildcat Field } \\
\text { Phillips Cnty, MT }\end{array}$ & $\begin{array}{l}\text { Phillips } \\
(1,600)\end{array}$ & 1,981 & & WF, D\&A. & \\
\hline $\begin{array}{l}\text { SP: } \\
\text { 6-30-79 } \\
\text { CP: } \\
7-31-79\end{array}$ & $\begin{array}{l}\text { J. J. C. Paine } \\
\text { and Associates }\end{array}$ & 1 Chapman & 13 & $\begin{array}{l}\text { csw 28/130N/ } \\
106 \mathrm{~W} \\
\text { Little Missouri } \\
\text { Field } \\
\text { Bowman Cnty, ND }\end{array}$ & $\begin{array}{l}\text { Eagle } \\
(1,215- \\
1.342)\end{array}$ & $\begin{array}{l}1.560 \\
\text { PB: } \\
1,420\end{array}$ & $\begin{array}{l}24,612 \mathrm{gal} \text { water. } \\
75,000 \mathrm{lb} \text { sand }\end{array}$ & $\begin{array}{l}\text { DG, no } \\
\text { cores or } \\
\text { tests, oper- } \\
\text { ator has } 4 \\
\text { additional } \\
\text { Eagle com- } \\
\text { pletions in } \\
\text { locale. }\end{array}$ & 368 \\
\hline
\end{tabular}


Table 2-4 Summary of Active Wells and New Locations - Northern Great Plains Province

\begin{tabular}{|c|c|c|c|c|c|c|}
\hline DATE & OPERATOR & WELL NAME & $\begin{array}{r}\text { MAP } \\
\text { NDE } \\
\text { NO }\end{array}$ & $\begin{array}{l}\text { LOCATION } \\
\text { Sec/T/R }\end{array}$ & $\begin{array}{l}\text { PROJECTED } \\
\text { HORIzON } \\
\text { (Depth in } \mathrm{ft} \text { ) }\end{array}$ & COMMENTS \\
\hline $\begin{array}{l}\text { FR: } \\
\text { 9-4-79 }\end{array}$ & $\begin{array}{l}\text { Sugar Creek } \\
\text { Resources }\end{array}$ & $\begin{array}{l}\text { 1-3 BN- } \\
\text { Pickard } \\
\text { Brothers }\end{array}$ & 1 & $\begin{array}{l}\text { nesw } 3 / 4 N / 61 \mathrm{E} \\
\text { Cedar Creek Field } \\
\text { Fallon Cty. MT }\end{array}$ & $\begin{array}{l}\text { Eagle } \\
(2,000)\end{array}$ & $\begin{array}{l}\text { D, operator has } 1 \text { other Eagle test in } \\
\text { this field. }\end{array}$ \\
\hline $\begin{array}{l}\text { FR: } \\
\text { 9-19-79 }\end{array}$ & Orlyn Terry & 28-1 Federal & 2 & $\begin{array}{l}\text { swsw } 28 / 7 \mathrm{~S} / 69 \mathrm{E} \\
\text { Wildcat Field } \\
\text { Carter Cty, MT }\end{array}$ & $\begin{array}{l}\text { Muddy } \\
(1,700)\end{array}$ & $\begin{array}{l}\text { WF, operator has } 2 \text { additional Muddy } \\
\text { tests in the area. }\end{array}$ \\
\hline $\begin{array}{l}\text { FR: } \\
\text { 7-30-79 }\end{array}$ & $\begin{array}{l}\text { Jerry } \\
\text { McCutchin, Jr. }\end{array}$ & $\begin{array}{l}2-16 \\
\text { Harrison- } \\
\text { State }\end{array}$ & 3 & $\begin{array}{l}\text { cse } 16 / 16 N / 3 E \\
\text { Cady Creek Field } \\
\text { Harding Cty, SD }\end{array}$ & $\begin{array}{l}\text { Eagle } \\
(2,800)\end{array}$ & $\begin{array}{l}\text { D, operator has } 1 \text { Greenhorn and } 5 \\
\text { Shannon tests in this area. }\end{array}$ \\
\hline $\begin{array}{l}\text { FR: } \\
7-20-79\end{array}$ & $\begin{array}{l}\text { J. Burns } \\
\text { Brown }\end{array}$ & $\begin{array}{l}17-15 \\
\text { Federal }\end{array}$ & 4 & $\begin{array}{l}\text { sese } 17 / 26 \mathrm{~N} / 16 \mathrm{E} \\
\text { Unnamed Field } \\
\text { Chouteau Cty, MT }\end{array}$ & $\begin{array}{l}\text { Eagle } \\
(\mathbf{2}, \mathbf{5 0 0})\end{array}$ & $\begin{array}{l}\text { D, operator has } 3 \text { additional Eagle } \\
\text { tests in this locale. }\end{array}$ \\
\hline $\begin{array}{l}\text { FR: } \\
\text { 8-13-79 } \\
\text { SP: } \\
\text { 8-11-79 }\end{array}$ & Balcron Oil & $\begin{array}{l}\text { 1-10 Van } \\
\text { Dyken }\end{array}$ & 5 & $\begin{array}{l}\text { sese } 10 / 29 N / 2 W \\
\text { Wildcat Field } \\
\text { Pondera Cty, MT }\end{array}$ & $\begin{array}{l}\text { Bow } \\
\text { Island } \\
(700)\end{array}$ & $\begin{array}{l}\text { WF, } 786 \text { TD. . . WOCT, operator } \\
\text { has } 4 \text {-other Bow Island tests in } \\
\text { Ledger Field. }\end{array}$ \\
\hline $\begin{array}{l}\text { FR: } \\
\text { 8-22-79 }\end{array}$ & Stroock et al & $\begin{array}{l}\text { Lisco \& } \\
\text { Stroock }\end{array}$ & 6 & $\begin{array}{l}\text { nesw } 30 / 30 \mathrm{~N} / 3 \mathrm{~W} \\
\text { Wildcat Field } \\
\text { Pondera Cty, MT }\end{array}$ & $\begin{array}{l}\text { Bow } \\
\text { Island } \\
(1,600)\end{array}$ & $\begin{array}{l}\text { WF, operator has } 1 \text { other Bow Island } \\
\text { test in this locale. }\end{array}$ \\
\hline $\begin{array}{l}\text { FR: } \\
\text { 6-26-79 }\end{array}$ & $\begin{array}{l}\text { Tricentrol } \\
\text { U.S. . }\end{array}$ & 10-10 Hofeldt & 7 & $\begin{array}{l}\text { nwse } 10 / 30 \mathrm{~N} / 18 \mathrm{E} \\
\text { Tiger Ridge Field } \\
\text { Blaine Cty, MT }\end{array}$ & $\begin{array}{l}\text { Eagle } \\
(1,350)\end{array}$ & $\begin{array}{l}\text { D, operator has } 6 \text { other Eagle tests } \\
\text { in this locale. }\end{array}$ \\
\hline $\begin{array}{l}\text { FR: } \\
2-19-79 \\
\text { SP: } \\
7-10-79\end{array}$ & $\begin{array}{l}\text { Montana } \\
\text { Dakota } \\
\text { Utilities }\end{array}$ & 966 Smith & 8 & $\begin{array}{l}\text { nenw } 13 / 31 N / 43 E \\
\text { Bowdoin Field } \\
\text { Valley Cty, MT }\end{array}$ & $\begin{array}{l}\text { Bowdoin- } \\
\text { Phillips } \\
(1,400)\end{array}$ & $\begin{array}{l}\text { D, 1,200 TD, } 1,166 \text { PB } \\
\ldots \text { WOCT. }\end{array}$ \\
\hline $\begin{array}{l}\text { FR: } \\
9-4-79\end{array}$ & $\begin{array}{l}\text { Marquis } \\
\text { Petroleum }\end{array}$ & $\begin{array}{l}26-1 \\
\text { McCarter }\end{array}$ & 9 & $\begin{array}{l}\text { nene } 26 / 32 N / 2 E \\
\text { Wildcat Field } \\
\text { Toole Cty, MT }\end{array}$ & $\begin{array}{l}\text { Bow } \\
\text { Island } \\
(1,200)\end{array}$ & $\begin{array}{l}\text { WF, operator has } 1 \text { additional Bow } \\
\text { Island test in the area. }\end{array}$ \\
\hline $\begin{array}{l}\text { FR: } \\
\text { 6-25-79 } \\
\text { SP: } \\
\text { 6-24-79 }\end{array}$ & $\begin{array}{l}\text { J. Burns } \\
\text { Brown }\end{array}$ & $\begin{array}{l}\text { 26-32-18 } \\
\text { Ramberg }\end{array}$ & 10 & $\begin{array}{l}\text { senw } 25 / 32 N / 18 E \\
\text { Black Coulee Field } \\
\text { Blaine Cty, MT }\end{array}$ & $\begin{array}{l}\text { Eagle } \\
(1,500)\end{array}$ & $\begin{array}{l}\text { D, Drlg ... no depth reported. } \\
\text { Operator has } 6 \text { other Eagle tests } \\
\text { in this locale. }\end{array}$ \\
\hline $\begin{array}{l}\text { FR: } \\
7-11-79\end{array}$ & $\begin{array}{l}\text { Falcon- } \\
\text { Colorado } \\
\text { Exploration }\end{array}$ & 1-27 Bell & 11 & $\begin{array}{l}\text { swsw } 27 / 33 N / 34 E \\
\text { Swanson Creek Field } \\
\text { Phlllps Cty, MT }\end{array}$ & $\begin{array}{l}\text { Phillips } \\
(1,700)\end{array}$ & $\begin{array}{l}\text { D, operator has } 5 \text { additional Phillips } \\
\text { tests in the area. }\end{array}$ \\
\hline $\begin{array}{l}\text { FR: } \\
\text { 8-21-79 }\end{array}$ & $\begin{array}{l}\text { Texas Oil } \\
\text { and Gas }\end{array}$ & $\begin{array}{l}\text { 1-S Leonard } \\
\text { A. Miller }\end{array}$ & 12 & $\begin{array}{l}\text { swsw } 20 / 34 N / 17 E \\
\text { Wildcat Field } \\
\text { Hill Cty, MT }\end{array}$ & $\begin{array}{l}\text { Eagle } \\
(1,700)\end{array}$ & $\begin{array}{l}\text { WF, operator has } 1 \text { other Eagle wild- } \\
\text { cat in the area. }\end{array}$ \\
\hline $\begin{array}{l}\text { FR: } \\
\text { 8-30-79 }\end{array}$ & $\begin{array}{l}\text { Webb } \\
\text { Resources }\end{array}$ & $\begin{array}{l}25-13 \\
\text { State }\end{array}$ & 13 & $\begin{array}{l}\text { swsw } 25 / 37 \mathrm{~N} / 4 \mathrm{~W} \\
\text { Fitzpatrick Lake } \\
\text { Field } \\
\text { Toole Cty, M T }\end{array}$ & $\begin{array}{l}\text { Bow } \\
\text { Island } \\
(1,860)\end{array}$ & $\begin{array}{l}\text { D, operator has } 4 \text { other Bow Island } \\
\text { tests in the area. }\end{array}$ \\
\hline $\begin{array}{l}\text { FR: } \\
\text { 6-4-79 } \\
\text { SP: } \\
\text { 8-26-79 }\end{array}$ & $\begin{array}{l}\text { J. J. C. Paine } \\
\text { \& Associates }\end{array}$ & 2-14 BLM & 14 & $\begin{array}{l}\text { cnw } 4 / 129 \mathrm{~N} / 106 \mathrm{~W} \\
\text { Little Missouri Field } \\
\text { Bowman Cty. ND }\end{array}$ & $\begin{array}{l}\text { Eagle } \\
(1,500)\end{array}$ & $\begin{array}{l}\text { D, 1,410 TD ... WOCT, operator } \\
\text { has } 10 \text { additional Eagle tests in } \\
\text { this locale. }\end{array}$ \\
\hline
\end{tabular}




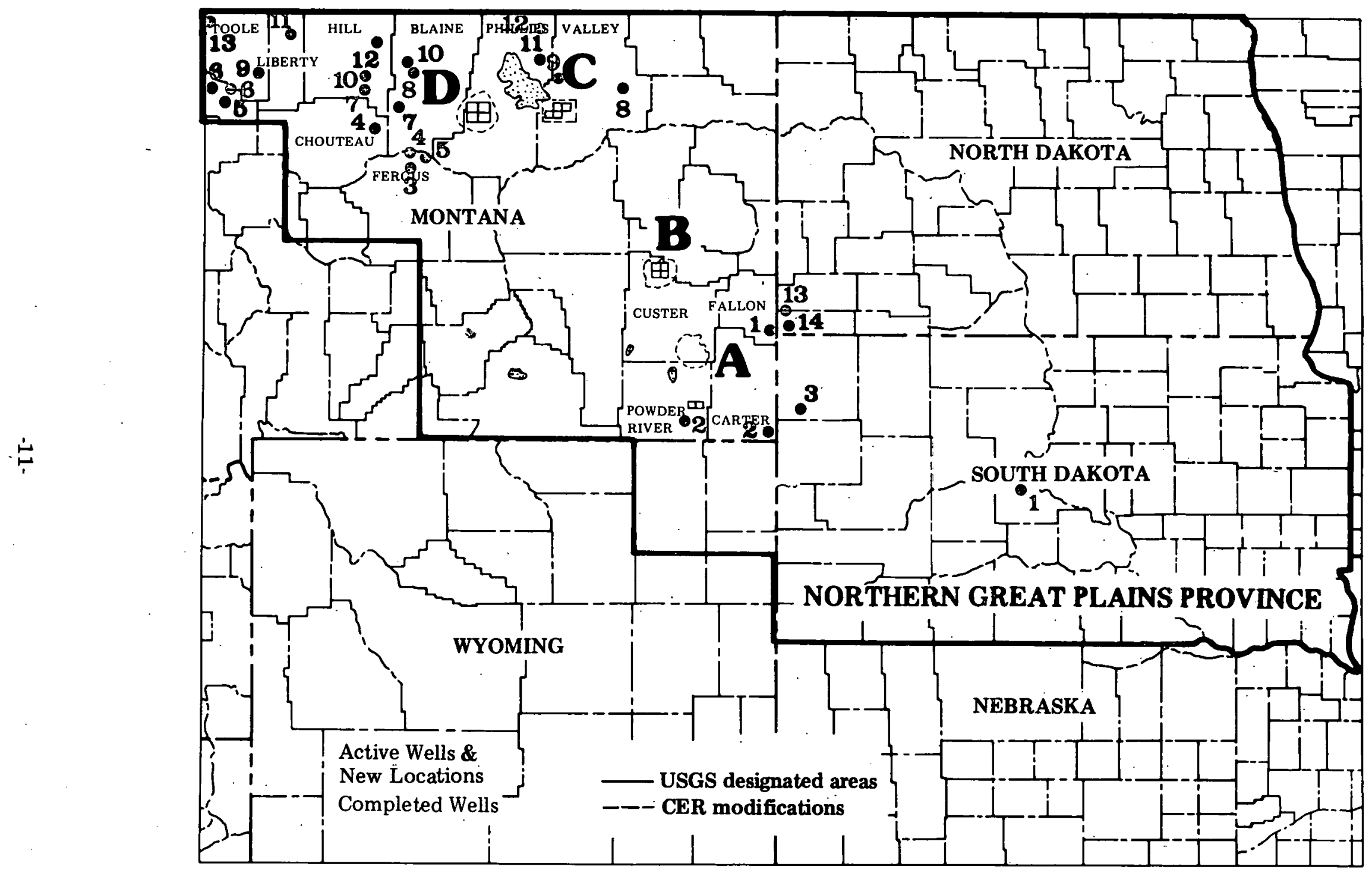

Figure 2-3 Wells Completed and Staked, USGS Designated Core Areas, Northern Great Plains Province (refer to Tables 2-3 and 2-4) 


\section{THIS PAGE}

\section{WAS INTENTIONALLY \\ LEFT BLANK}




\section{Greater Green River Basin}

\subsection{CORE PROGRAM}

To assist in assessing the resource potential within the Greater Green River Basin, the USGS recommended core acquisition in the following areas (see Figure 3-1):

\section{Area A}

The crest of the Wamsutter Arch in Sweetwater and Carbon Counties, the Northern edge bordering on the Red Desert Basin and the southern edge occupying part of the Washakie Basin, $\mathrm{T} 17 \mathrm{~N}$ to $\mathrm{T} 21 \mathrm{~N}$, and $\mathrm{R} 91 \mathrm{~W}$ to $\mathrm{R} 97 \mathrm{~W}$. The section below the commercial Almond and Ericson (Mesaverde) is of primary interest in this area.

\section{Area B}

The area east of the Big Piney/LaBarge Field in Sweetwater and Sublette Counties running north and south through the Pinedale area and to the northern edge of the Green River Basin. These areas skirt the edge of the Wind River Range. Production here is primarily Frontier, with some contribution from the Mesaverde and Fort Union.

Area $\mathrm{B}$ has been divided into four subdivisions which are:

$B_{1}$ West of R104W to R109W and from T22N, north to T28N

$\mathrm{B}_{2} \mathrm{R} 106 \mathrm{~W}$ to $\mathrm{R} 111 \mathrm{~W}$ and from $\mathrm{T} 27 \mathrm{~N}$ to $\mathrm{T} 31 \mathrm{~N}$

$B_{3}$ R108W to R112W but not including development drilling, and T30N to T35N

$B_{4}$ North edge of Green River Basin; north into the Hoback Basin, R112W to R114W and $\mathrm{T} 36 \mathrm{~N}$ to $\mathrm{T} 39 \mathrm{~N}$. 


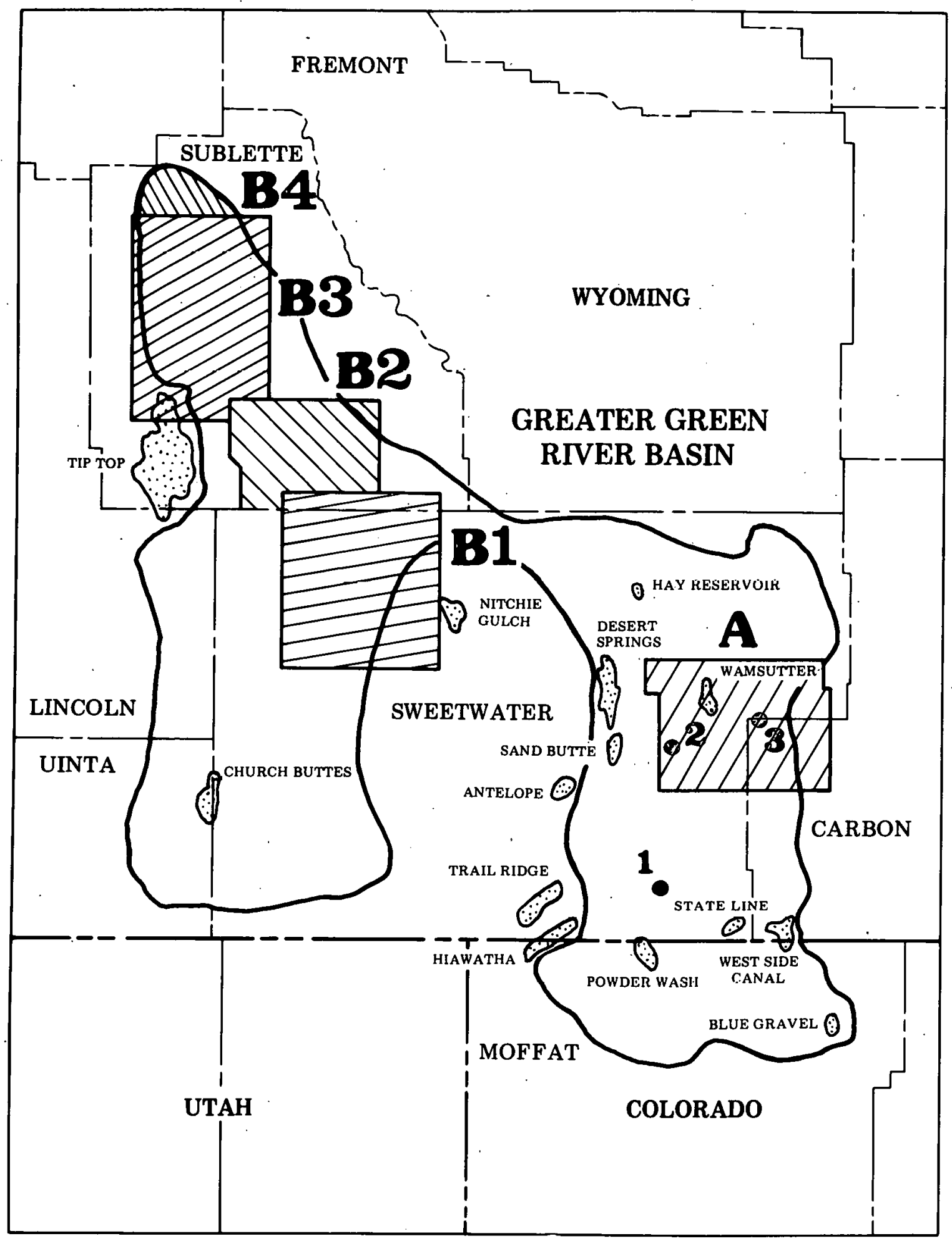

Figure 3-1 USGS Designated Core Areas and Wells of Interest, Greater Green River Basin (refer to Table 3-1) 
The core sites contain thick sections of tight, but generally untested gas sands, in Upper Cretaceous and Tertiary formations.

In the Greater Green River Basin, Colorado Interstate Gas Exploration was contacted, but declined the coring of their Haystack Unit No. 2-27-14-96 well in Sweetwater County, Wyoming. Also in Sweetwater County, Pacific Transmission Supply Company has been contacted in regards to coring their well No. 3-10A Federal in the Red Desert sub-basin and a contract is pending. Cotton Petroleum turned down a coring proposal for wells in Sweetwater County because of possible high pressures, but will be interested in future proposals. Yates Petroleum declined a proposal, but would like to discuss future wells. Texas Oil and Gas is considering a proposal. Table 3-1 is a summary of wells located in the core areas and Figure 3-1 shows the location of these wells.

\begin{tabular}{|c|c|c|c|c|c|c|c|c|c|}
\hline \multirow{3}{*}{$\begin{array}{l}\text { DATE } \\
\text { FR: } \\
\text { 6-26-79 }\end{array}$} & \multirow{3}{*}{$\begin{array}{l}\text { Table 3-1 } \\
\text { OPERATOR } \\
\begin{array}{l}\text { CIG } \\
\text { Exploration }\end{array}\end{array}$} & \multicolumn{8}{|c|}{$\begin{array}{l}\text { Summary of Wells Located in USGS Designated Core Areas - } \\
\text { Greater Green River Basin }\end{array}$} \\
\hline & & WELL NAME & $\begin{array}{c}\text { MAP } \\
\text { INDEX } \\
\text { NO. }\end{array}$ & $\begin{array}{l}\text { LOCATION (Px } \\
\text { Sec/T/R or Pre }\end{array}$ & $\begin{array}{l}\text { HORIZON } \\
\text { rojected Depth } \\
\text { oducing Interval) }\end{array}$ & $\begin{array}{c}\text { FINAL } \\
\text { TD }\end{array}$ & $\begin{array}{l}\text { FRACTURE } \\
\text { TREATMENT }\end{array}$ & COMMENTS & $\begin{array}{l}\text { IPF in } \\
\text { MCFD }\end{array}$ \\
\hline & & $\begin{array}{l}2-27-14-96 \\
\text { Haystack Unit }\end{array}$ & 1 & $\begin{array}{l}\text { sene } 17 / 14 \mathrm{~N} / 96 \mathrm{~W} \\
\text { Haystack Field } \\
\text { Sweetwater Cnty, } \\
\text { WY }\end{array}$ & $\begin{array}{l}\text { Mesaverde } \\
(16,500)\end{array}$ & & . & $\begin{array}{l}\text { Wo, operator } \\
\text { has } 1 \text { addi- } \\
\text { tional Mesa- } \\
\text { verde test in } \\
\text { locale. }\end{array}$ & \\
\hline $\begin{array}{l}\text { FR: } \\
\text { 12-4-78 }\end{array}$ & Davis Oll & $\begin{array}{l}1 \text { Coronet } \\
\text { Federal }\end{array}$ & 2 & $\begin{array}{l}\text { cse } 8 / 18 N / 92 W \\
\text { Creston Fleld } \\
\text { Carbon Cnty, WY }\end{array}$ & $\begin{array}{l}\text { Mesaverde } \\
(9,800)\end{array}$ & & & $\begin{array}{l}\text { wo, sus, located } \\
\text { in Core Area } A . \\
\text { Operator has } 3 \\
\text { additional wells in } \\
\text { locale. }\end{array}$ & \\
\hline $\begin{array}{l}\text { FR: } \\
\text { 6-26-79 }\end{array}$ & $\begin{array}{l}\text { Amoco } \\
\text { Production }\end{array}$ & $\begin{array}{l}9-7 \text { Tierney } \\
\text { II Unit }\end{array}$ & 3 & $\begin{array}{l}\operatorname{csw} 7 / 19 N / 93 W \\
\text { Wudcat Field } \\
\text { Carbon Cnty. WY }\end{array}$ & $\begin{array}{l}\text { Mesaverde } \\
(10.155)\end{array}$ & & & $\begin{array}{l}\text { WF. located in } \\
\text { Core Area A. } \\
\text { Operator has } \\
35 \text { additional } \\
\text { wells in imme- } \\
\text { diate area. }\end{array}$ & \\
\hline $\begin{array}{l}\text { FR: } \\
\text { 8-29-79 }\end{array}$ & $\begin{array}{l}\text { Amoco } \\
\text { Production }\end{array}$ & $\begin{array}{l}1 \text { Champlin- } \\
201 \text { Amoco-B }\end{array}$ & 4 & $\begin{array}{l}\text { w/saw } 9 / 21 \mathrm{~N} / 95 \mathrm{~W} \\
\text { Wildcat Field } \\
\text { Sweetwater Cnty. } \\
\text { WY }\end{array}$ & $\begin{array}{l}\text { Mesaverde } \\
(10.600)\end{array}$ & & . & $\begin{array}{l}\text { WF, located in } \\
\text { Core Area A. } \\
\text { Operator has } \\
\text { 3 additional } \\
\text { wildcat wells } \\
\text { in area. }\end{array}$ & \\
\hline $\begin{array}{l}\text { FR: } \\
\text { 6-25-79 }\end{array}$ & $\begin{array}{l}\text { Pacific } \\
\text { Transmission } \\
\text { Supply }\end{array}$ & 3-1 2 Federal & 5 & $\begin{array}{l}\text { nwsw } 12 / 22 N / 92 W \\
\text { Wildcat Field } \\
\text { Sweet water Cnty. } \\
\text { WY }\end{array}$ & $\begin{array}{l}\text { Mesaverde } \\
(13,550)\end{array}$ & & . & $\begin{array}{l}\text { WF, located in } \\
\text { Core Area A. } \\
\text { Operator has } 3 \\
\text { additional wild- } \\
\text { cat wells in area. }\end{array}$ & \\
\hline $\begin{array}{l}\text { SP: } \\
11 \cdot 18 \cdot 78 \\
\text { CP: } \\
7-10-79\end{array}$ & $\begin{array}{l}\text { Energetics } \\
\text { Incorporated } \\
.\end{array}$ & 10.29 Federal & $\dot{6}$ & $\begin{array}{l}\text { nwnw } 29 / 27 N / \\
111 w \\
\text { Bird Canyon Field } \\
\text { Sublette Cnty. WY }\end{array}$ & $\begin{array}{l}\text { Bear River } \\
(9,693-9.730)\end{array}$ & $\begin{array}{l}9.756 \\
\text { PB: } \\
9,874 \\
\quad .\end{array}$ & $\begin{array}{l}64,700 \mathrm{gal} \mathrm{gel} \\
79,000 \mathrm{lb} \text { sand }\end{array}$ & $\begin{array}{l}\text { DG, no cores } \\
\text { or tests. Located } \\
\text { in Core Area B2. } \\
\text { Operator has } 5 \\
\text { additional wells } \\
\text { in immediate } \\
\text { locale. }\end{array}$ & 4.400 \\
\hline
\end{tabular}


Table 3-2 Summary of Drilling Activities, Greater Green River Basin *

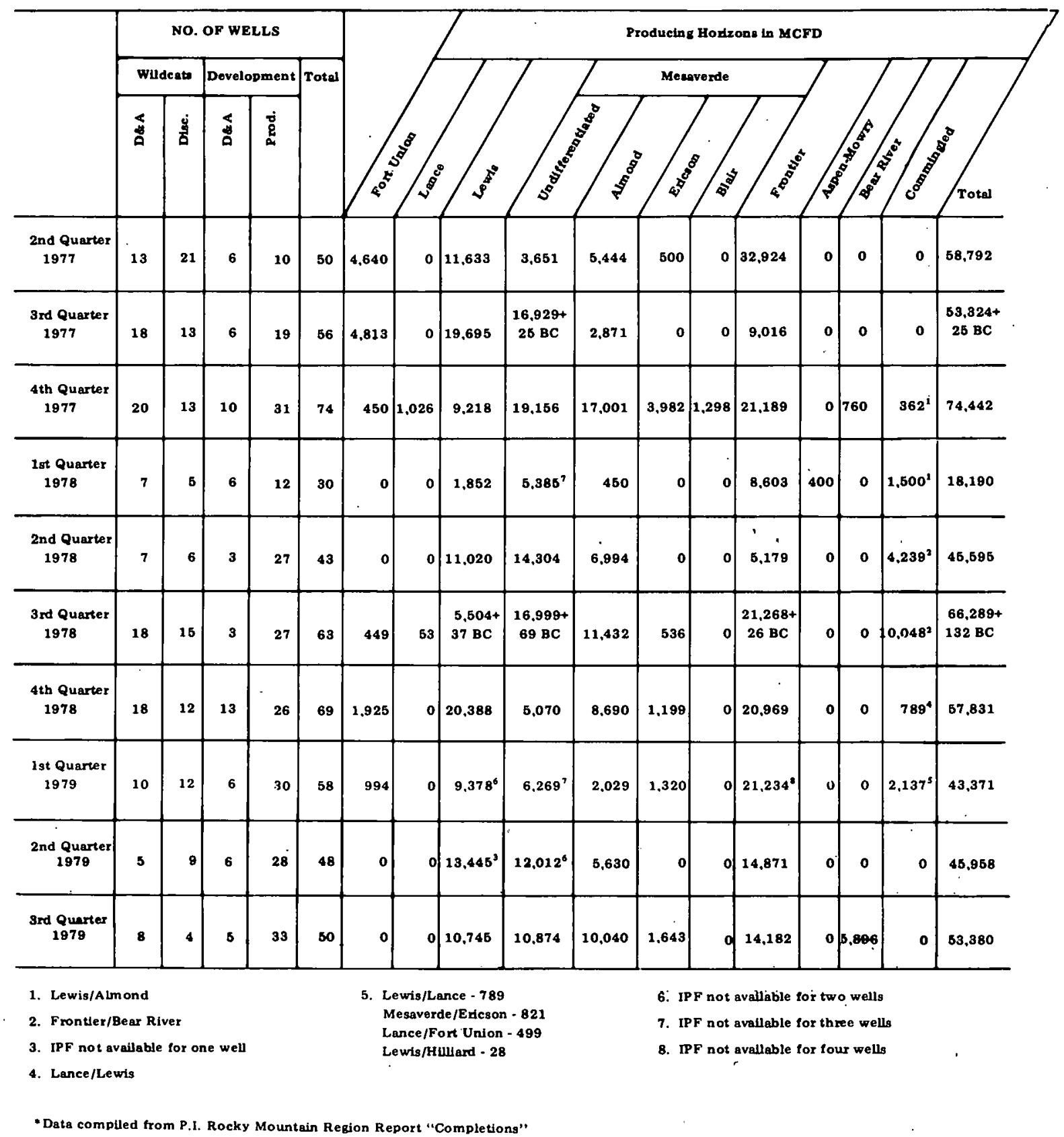




\subsection{DRILLING ACTIVITIES}

In this quarter, new gas production from Upper Cretaceous and Tertiary horizons totalled 53,380 MCFD, up 14 percent from last quarter. The Frontier Formation contributed 27 percent of the new gas for the quarter, the Lewis Formation and undifferentiated Mesaverde Group contributed 20 percent each, the Almond produced 19 percent of the total, the Bear River produced 11 percent and the Ericson contributed 3 percent.

Fifty wells were completed this quarter. Thirty-three new development wells were producing by the quarter's end, with 5 wells D\&A. Four wildcat wells were producers and 8 were D\&A. The producing horizons and a summary of drilling activities are listed in Table 3-2. Summaries of Greater Green River completed wells, active and newly located wells appear in Tables 3-3 and 3-4, respectively. Figure 3-2 shows the locations of these wells.

Table 3-3 Summary of Wells Completed-Greater Green River Basin

\begin{tabular}{|c|c|c|c|c|c|c|c|c|c|}
\hline DATE & OPERATOR & WELL NAME & $\begin{array}{l}\text { MAP } \\
\text { INDEX } \\
\text { NO. }\end{array}$ & $\begin{array}{l}\text { LOCATION } \\
\text { Sec/T/R }\end{array}$ & $\begin{array}{l}\text { HORIzON } \\
\text { cofected Depth } \\
\text { oduding Interval) }\end{array}$ & $\underset{\text { TD }}{\text { FTNAL }}$ & $\begin{array}{l}\text { FRACTURE } \\
\text { TREATMENT }\end{array}$ & COMMENTS & $\begin{array}{l}\text { IPF in } \\
\text { MCFD }\end{array}$ \\
\hline $\begin{array}{l}\text { SP: } \\
\text { G-1-79 } \\
\text { CP: } \\
7-28-79\end{array}$ & Sun Gas & $\begin{array}{l}\text { 29-1 Federal- } \\
\text { Fox }\end{array}$ & 1 & $\begin{array}{l}\text { nwnw } 29 / 8 \text { N/90w } \\
\text { Craig North Field } \\
\text { Moffat Cnty, Co }\end{array}$ & $\begin{array}{l}\text { Lewis } \\
(2,918-3,277)\end{array}$ & 3.630 & $\begin{array}{l}37,100 \mathrm{gal} \mathrm{emul} \\
64,000 \mathrm{lb} \text { sand }\end{array}$ & DG & 312 \\
\hline $\begin{array}{l}\text { SP: } \\
8-4-78 \\
\text { CP: } \\
8-20-78\end{array}$ & $\begin{array}{l}\text { CIG } \\
\text { Exploration }\end{array}$ & $\begin{array}{l}1-2-8-91 \\
\text { Visintainer } \\
\text { Sheep }\end{array}$ & 2 & $\begin{array}{l}\text { nesw 2/8N/91w } \\
\text { Blue Gravel Field } \\
\text { Motfat Cnty, co }\end{array}$ & 5,200 test & 4.807 & & $\begin{array}{l}\text { Wo, D\&A, no } \\
\text { cores. }\end{array}$ & \\
\hline $\begin{array}{l}\text { SP: } \\
7-9-79 \\
\text { CP: } \\
7-21-79\end{array}$ & $\begin{array}{l}\text { Anadarko } \\
\text { Production }\end{array}$ & $\begin{array}{l}1 \text { Cottonwood } \\
\text { Gulch-Federal- } \\
\text { B }\end{array}$ & 2 & $\begin{array}{l}\text { nene 26/8N/91W } \\
\text { North Craig Field } \\
\text { Moftat Cnty, CO }\end{array}$ & $\begin{array}{l}\text { Lewis } \\
(3,900)\end{array}$ & 4,017 & & $\begin{array}{l}\text { D, D\& A, operator } \\
\text { has } 7 \text { additional } \\
\text { wells in locale. }\end{array}$ & \\
\hline $\begin{array}{l}\text { FR: } \\
7-11-79\end{array}$ & $\begin{array}{l}\text { Cotton } \\
\text { Petroleum }\end{array}$ & $\begin{array}{l}\text { 18-1 Hammond } \\
\text { Federal }\end{array}$ & 3 & 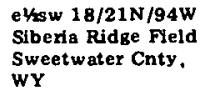 & $\begin{array}{l}\text { Mesavende } \\
(10.600)\end{array}$ & & . & D, abn location. & . \\
\hline $\begin{array}{l}\text { SP: } \\
2-13-77 \\
\text { CP: } \\
\text { 5-17-79 }\end{array}$ & $\begin{array}{l}\text { Amoco } \\
\text { Production }\end{array}$ & $\begin{array}{l}1 \text { Champlin } \\
336 \text { Amoco-A }\end{array}$ & 4 & $\begin{array}{l}\text { nesw } 21 / 17 N / 94 W \\
\text { Wudcat Field } \\
\text { Sweetwater Cnty, } \\
\text { WY }\end{array}$ & $\begin{array}{l}\text { Mesaverde } \\
(12,800)\end{array}$ & 11,656 & $\begin{array}{l}\text { Acidized } 3 \text { times- } \\
\text { total } 227,926 \mathrm{gal} \text {, } \\
\text { tract } t \text { wice, total } \\
464,205 \mathrm{gal} \text { emul } \\
1,034,334 \mathrm{lb} \\
\text { sand }\end{array}$ & $\begin{array}{l}\text { WF, D\& A, opera- } \\
\text { tor has } 10 \text { addi- } \\
\text { tional wells in } \\
\text { immediate locale. } \\
\text { Largest tracture } \\
\text { reported in quarte }\end{array}$ & $\begin{array}{l}\text { a. } \\
\text { e. } \\
\text { ter. }\end{array}$ \\
\hline $\begin{array}{l}\text { SP: } \\
1-17-79 \\
\text { CP: } \\
\text { 4-22-79 }\end{array}$ & $\begin{array}{l}\text { C\&K } \\
\text { Petroleum }\end{array}$ & 1 Flying w & 5 & $\begin{array}{l}\text { nwne } 26 / 21 N / \\
113 W \\
\text { Widcat Field } \\
\text { Lincoln Cnty, WY }\end{array}$ & $\begin{array}{l}\text { Frontier } \\
\text { test } \\
(11,360)\end{array}$ & 11,593 & & WF, D\&A. & \\
\hline $\begin{array}{l}\text { SP: } \\
2-28-79 \\
\text { CP: } \\
7-31-79\end{array}$ & Davis Oll & $\begin{array}{l}\text { 3 Picket Lake } \\
\text { Unit }\end{array}$ & 6 & $\begin{array}{l}\text { nesw } 18 / 26 \text { N } / 96 W \\
\text { Picket Lake Field } \\
\text { Sweetwater Cnty. } \\
\text { WY }\end{array}$ & $\begin{array}{l}\text { Lewis } \\
(13,716- \\
13,736)\end{array}$ & 14,000 & $\begin{array}{l}178,650 \mathrm{gal} \text { emul } \\
191,250 \mathrm{lb} \text { sand }\end{array}$ & $\begin{array}{l}\text { WOE, Lewis } \\
\text { Extension- } \\
\text { Picket Lake. } \\
\text { Operator has } \\
7 \text { additional } \\
\text { wells in imme- } \\
\text { diate locale. }\end{array}$ & $\mathbf{3 , 1 7 4}$ \\
\hline $\begin{array}{l}\text { SP: } \\
\text { B-16-78 } \\
\text { CP: } \\
12-18-78\end{array}$ & $\begin{array}{l}\text { Belco } \\
\text { Petroleum }\end{array}$ & 26-9 Unit & 7 & $\begin{array}{l}\text { nwsw } 9 / 27 N / 113 w \\
\text { North Labarge } \\
\text { Field } \\
\text { Sublette Cnty, wY }\end{array}$ & $\begin{array}{l}\text { Frontiex } \\
(6,078-6,717)\end{array}$ & 6,784 & $\begin{array}{l}\text { Acidized } w / 2,500 \\
\text { gal }\end{array}$ & $\begin{array}{l}\text { DG, no cores } \\
\text { or tests. Opera- } \\
\text { tor has } 13 \text { addi- } \\
\text { tional drillings } \\
\text { in locale. }\end{array}$ & 244 \\
\hline
\end{tabular}




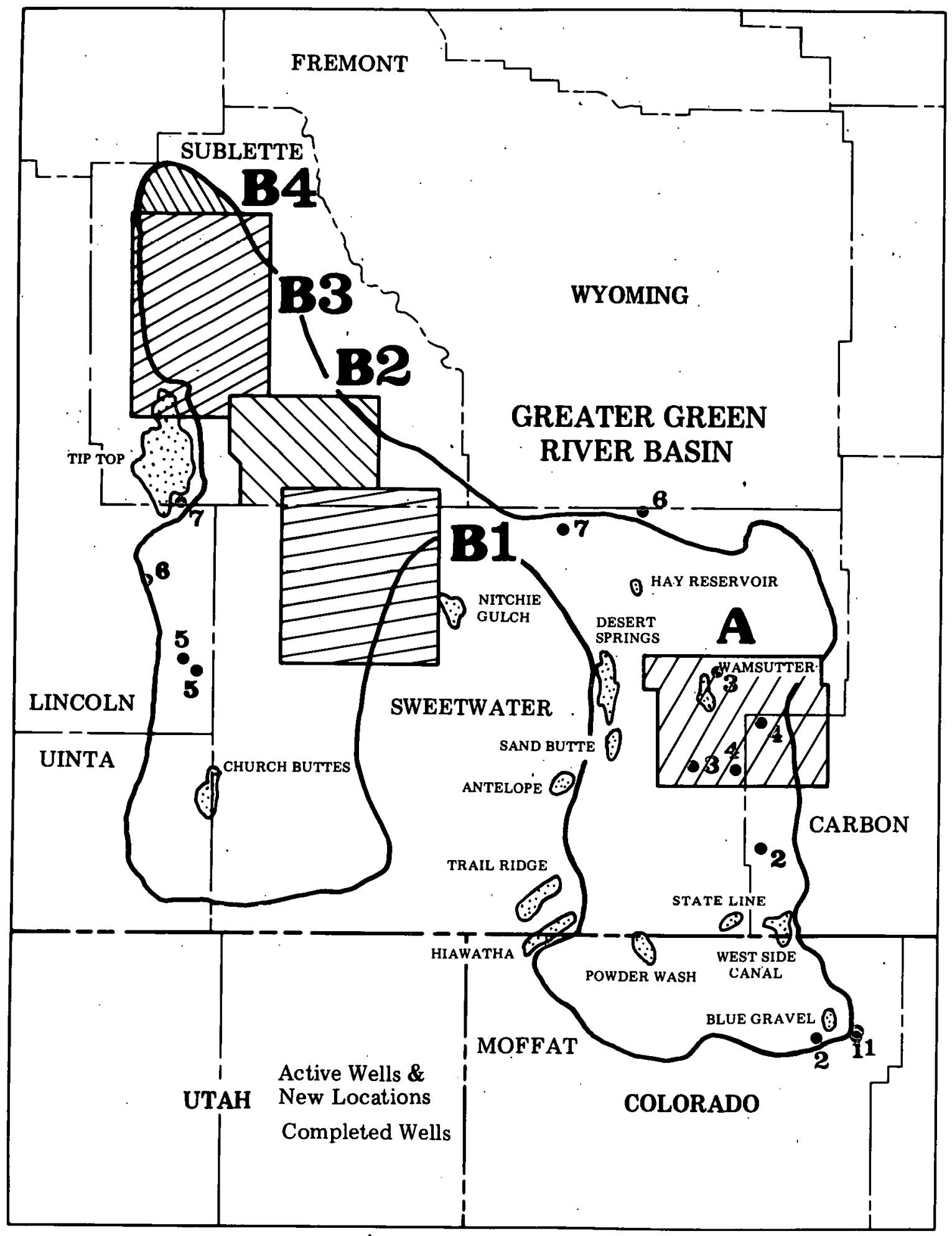

Figure 3-2 Wells Completed and Staked, USGS Designated Core Areas, Greater Green River Basin (refer to Tables 3-3 and 3-4) 
Table 3-4 Summary of Active Wells and New Locations-Greater Green River Basin

\begin{tabular}{|c|c|c|c|c|c|c|}
\hline DATE & OPERATOR & WELL NAME & $\begin{array}{l}\text { MAP } \\
\text { INDEP } \\
\text { NO. }\end{array}$ & $\begin{array}{c}\text { LOCATION } \\
\operatorname{Sec} / T / R\end{array}$ & $\begin{array}{l}\text { PROJECTED } \\
\text { HORIZON } \\
\text { (Depth in } \mathrm{ft} \text { ) }\end{array}$ & COMMENTS \\
\hline $\begin{array}{l}\text { FR: } \\
9-17-79\end{array}$ & Conoco Inc. & 1 Government & 1 s & $\begin{array}{l}\text { sw'sw } 21 / 9 \mathrm{~N} / 89 \mathrm{~W} \\
\text { Wildcat Field } \\
\text { Moffat Cnty, CO }\end{array}$ & $\begin{array}{l}\text { Lewis } \\
(4,165)\end{array}$ & $\begin{array}{l}\text { WF, operator has } 1 \text { additional wildcat } \\
\text { well in area, completed D\&A. }\end{array}$ \\
\hline $\begin{array}{l}\text { FR: } \\
\text { 9-4-79 }\end{array}$ & Sinclair Oil & $\begin{array}{l}12 \text { Blue Gap } \\
\text { II Unit }\end{array}$ & 2 & $\begin{array}{l}\text { nesw } 17 / 15 N / 93 W \\
\text { Wildcat Field } \\
\text { Carbon Cnty, WY }\end{array}$ & $\begin{array}{l}\text { Mesaverde } \\
(11,500)\end{array}$ & $\begin{array}{l}\text { WF, operator has } 4 \text { additional } \\
\text { development wells in locale. }\end{array}$ \\
\hline $\begin{array}{l}\text { FR: } \\
\text { 5-29-79 } \\
\text { SP: } \\
\text { 8-11-79 }\end{array}$ & $\begin{array}{l}\text { Amoco } \\
\text { Production }\end{array}$ & $\begin{array}{l}1 \text { USA- } \\
\text { Amoco-U }\end{array}$ & 3 & $\begin{array}{l}\text { swne } 18 / 17 \mathrm{~N} / 95 \mathrm{~W} \\
\text { Wildcat Field } \\
\text { Sweetwater Cnty, } \\
\text { WY }\end{array}$ & $\begin{array}{l}\text { Mesaverde } \\
(12,700)\end{array}$ & $\begin{array}{l}\text { WF, TD } 11,800 \text {, fishing. Operator } \\
\text { has } 9 \text { additional tests in the area. }\end{array}$ \\
\hline $\begin{array}{l}\text { FR: } \\
\text { 5-8-79 } \\
\text { SP: } \\
\text { 9-3-79 }\end{array}$ & $\begin{array}{l}\text { Pacific } \\
\text { Transmission } \\
\text { Supply }\end{array}$ & $\begin{array}{l}3-10 \\
\text { Federal }\end{array}$ & 4 s & $\begin{array}{l}\text { sesw } 10 / 19 \mathrm{~N} / 93 \mathrm{~W} \\
\text { Echo Springs Field } \\
\text { Carbon Cnty, WY }\end{array}$ & $\begin{array}{l}\text { Mesaverde } \\
(9,725)\end{array}$ & $\begin{array}{l}\text { D, drlg @ 7,428 ft. Operator has } \\
5 \text { additional Mesaverde tests in this } \\
\text { locale. }\end{array}$ \\
\hline $\begin{array}{l}\text { FR: } \\
\text { 5-11-79 } \\
\text { SP: } \\
7-3-79\end{array}$ & $\begin{array}{l}\text { Marathon } \\
\text { Oil }\end{array}$ & 15 Unit & 5 & $\begin{array}{l}\text { nesw } 32 / 20 \mathrm{~N} / 112 \mathrm{~W} \\
\text { Wilson Ranch Field } \\
\text { Lincoln Cnty, WY }\end{array}$ & $\begin{array}{l}\text { Frontier } \\
(11,350)\end{array}$ & $\begin{array}{l}\text { D, TD } 11,490, \text { wOCT. Operator } \\
\text { has } 2 \text { additional Frontier tests in } \\
\text { locale. }\end{array}$ \\
\hline $\begin{array}{l}\text { FR: } \\
\text { 9-17-79 }\end{array}$ & $\begin{array}{l}\text { Rainbow } \\
\text { Resources }\end{array}$ & 1-10 Federal & $6 \mathrm{r}$ & $\begin{array}{l}\text { nesw } 10 / 23 N / 114 W \\
\text { Wildcat Field } \\
\text { Lincoln Cnty, WY }\end{array}$ & $\begin{array}{l}\text { Hilliard } \\
(6,500)\end{array}$ & $\begin{array}{l}\text { WF, operator has one additional } \\
\text { Hilliard well in locale. }\end{array}$ \\
\hline $\begin{array}{l}\text { FR : } \\
9-18-79\end{array}$ & $\begin{array}{l}\text { Woods } \\
\text { Petroleum }\end{array}$ & $\begin{array}{l}3 \text { Lost } \\
\text { Valley Unit }\end{array}$ & 7 & $\begin{array}{l}\text { nesw } 20 / 25 \mathrm{~N} / 99 \mathrm{~W} \\
\text { Wildcat Field } \\
\text { Sweetwater Cnty, } \\
\text { WY }\end{array}$ & $\begin{array}{l}\text { Mesaverde } \\
(13,000)\end{array}$ & $\begin{array}{l}\text { WF, operator has } 3 \text { additional wells } \\
\text { in immediate locale. }\end{array}$ \\
\hline
\end{tabular}

The largest amount of drilling activity in the Greater Green River Basin is located east of the Rock Springs Uplift in and on the flanks of the Washakie and Red Desert sub-basins. Although the Mesaverde Group continues to command the most WGSP interest, younger units are the objectives in the deeper parts of the sub-basins, including sands in the Lewis and some Tertiary sections. The Wamsutter Arch, separating the Washakie and Red Desert sub-basins, and the Cherokee Ridge, separating the Washakie and Sand Wash sub-basins, are still active areas for exploration. The Mesaverde is the primary producer in the Wamsutter Arch, and the Tertiary, Wasatch and Ft. Union are the primary producers for the Cherokee Arch. Amoco Production, Kemmerer Coal, Pacific Transmission Supply Company, Getty Oil and Davis Oil are some of the most active companies with drilling objectives of interest to the WGSP. In Colorado's Sand Wash sub-basin, which is of interest to the WGSP, Tenneco Oil, Davis Oil, Northwest Exploration and Champlin Petroleum are seeking Lewis and Mesaverde production. The Green River Basin Proper has not been particularly active for the WGSP. Most activity has been out of the basin with Frontier as the objective section. 
Rainbow Resources is active on the north end of the Rock Springs Uplift, which separates the Red Desert from the Green River sub-basin. This is also an area of interest to the WGSP for Tertiary and Mesaverde section. Figure 3-3 is a stratigraphic correlation chart of the Greater Green River, Piceance and Uinta Basins.

The largest fracture job reported for the quarter was performed on Amoco Production's 1 Champlin 336 Amoco-A well, Sec. 21, T17N, R94W, Wildcat Field, Sweetwater County. The well was completed D\&A after being acidized three times with a total of 227,925 gal and fractured twice with a total of $464,205 \mathrm{gal}$ of emulsion and $1,034,334 \mathrm{lb}$ of sand. 


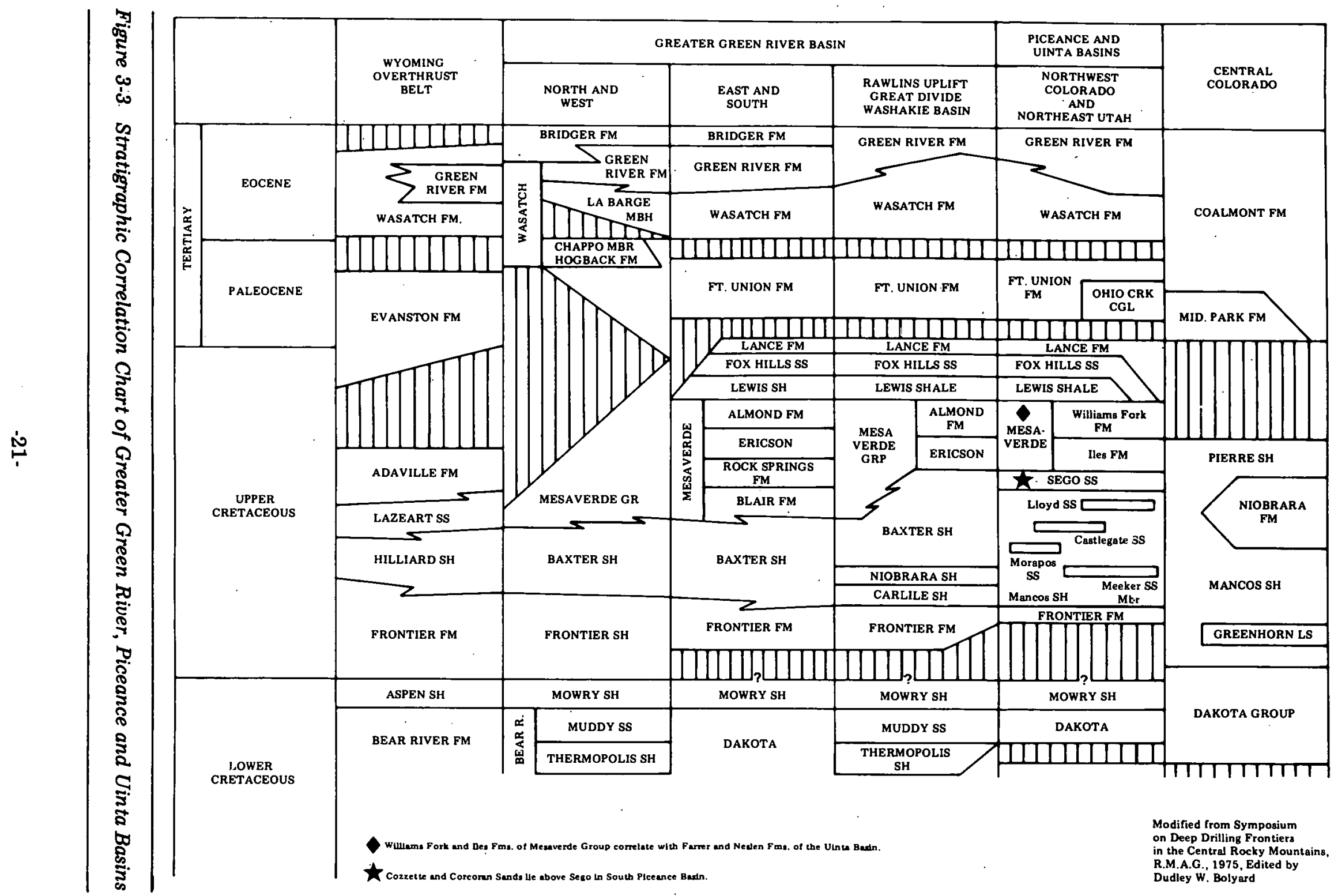


THIS PAGE

\section{WAS INTENTIONALLY \\ LEFT BLANK}




\section{Uinta Basin}

\subsection{CORE PROGRAM}

The Uinta Basin is located in northeastern Utah and has potential production from tight gas sands in a thick section of Upper Cretaceous through Tertiary sediments. Cretaceous tight horizons include the Castlegate and Sego sandstones and the Blackhawk, Price River, Neslen and Farrer Formations. In the Tertiary, tight gas sands are found in the Tuscher, North Horn, Wasatch and Green River Formations.

Areas recommended by the USGS for the acquisition of core for the WGSP core program are listed below (See Figure 4-1).

\section{Area A}

Wasatch and Duchesne Counties - T4S to T7S, R5W to R11W; in Wasatch County, T9S to T11S, R7E to R9E; in Carbon County, T11S and T12S, R7E to R13E.

Area B

Duchesne and Uintah Counties - T3S to T4S, R2E to R2W and parts of T8S, R16N to $\mathrm{R} 18 \mathrm{~W}$.

Area C

Uintah County - T8S and T9S and R23E to R25E.

\section{Area D}

Uintah and Grand Counties - T10S, R18E, T11S to T16S, R18E to R25E; and in T17S to T19S, R21E and R22E. 
These areas were selected due to their lack of core and well data, and location outside the main productive fields of the Uinta Basin. Activity is concentrated in and around the Natural Buttes and Red Wash Fields, where production is primarily from the Green River and Wasatch Formations, and Mesaverde Group.

Within the core areas, newly staked locations are monitored so that operators can be contacted concerning participation in the WGSP core program. Burton-Hawks and Chandler Resources declined core proposals for the Uinta Basin. Mapco, Inc. was contacted and discussions continued concerning the coring of a Wasatch-Mesaverde interval from Mapco RBU No. 11-17F well, Sec. 17, T10S; R20E, Uinta County, Utah. Spud in is expected around mid-October, with coring planned around the first of November.

To supplement outcrop studies, a 6-1/2 in. diameter core hole (DOE/WGS-GC-1) is being drilled by $\mathrm{C} \mathrm{K}$ GeoEnergy through about $1,200 \mathrm{ft}$ of the non-marine Mesaverde Group in the Westwater Canyon area of the Book Cliffs in eastern Utah (Sec. 16, T17S, R24E, Grand County). The core hole is located on a ridge and the section being cored (lower Tuscher, Farrer, Upper Neslen), is exposed along the adjacent valley walls. There was excellent core recovery and $4,400 \mathrm{lbs}$ of $3 \mathrm{in}$. diameter core was shipped to the USGS in Denver for description. An additional $600 \mathrm{ft}$ of core will be cut and the hole logged. This work is being done under contract to DOE for characterization of lenticular sands.

Table 4-1 is a summary of Uinta Basin core area wells and Figure 4-1 shows their locations.

\subsection{DRILLING ACTIVITIES}

Twelve development wells were reported completed during the third quarter of 1979 in Upper Cretaceous and Tertiary horizons of interest to the WGSP. There was no wildcat activity. All twelve development wells were producers. (Abandoned locations are not listed in this report). 


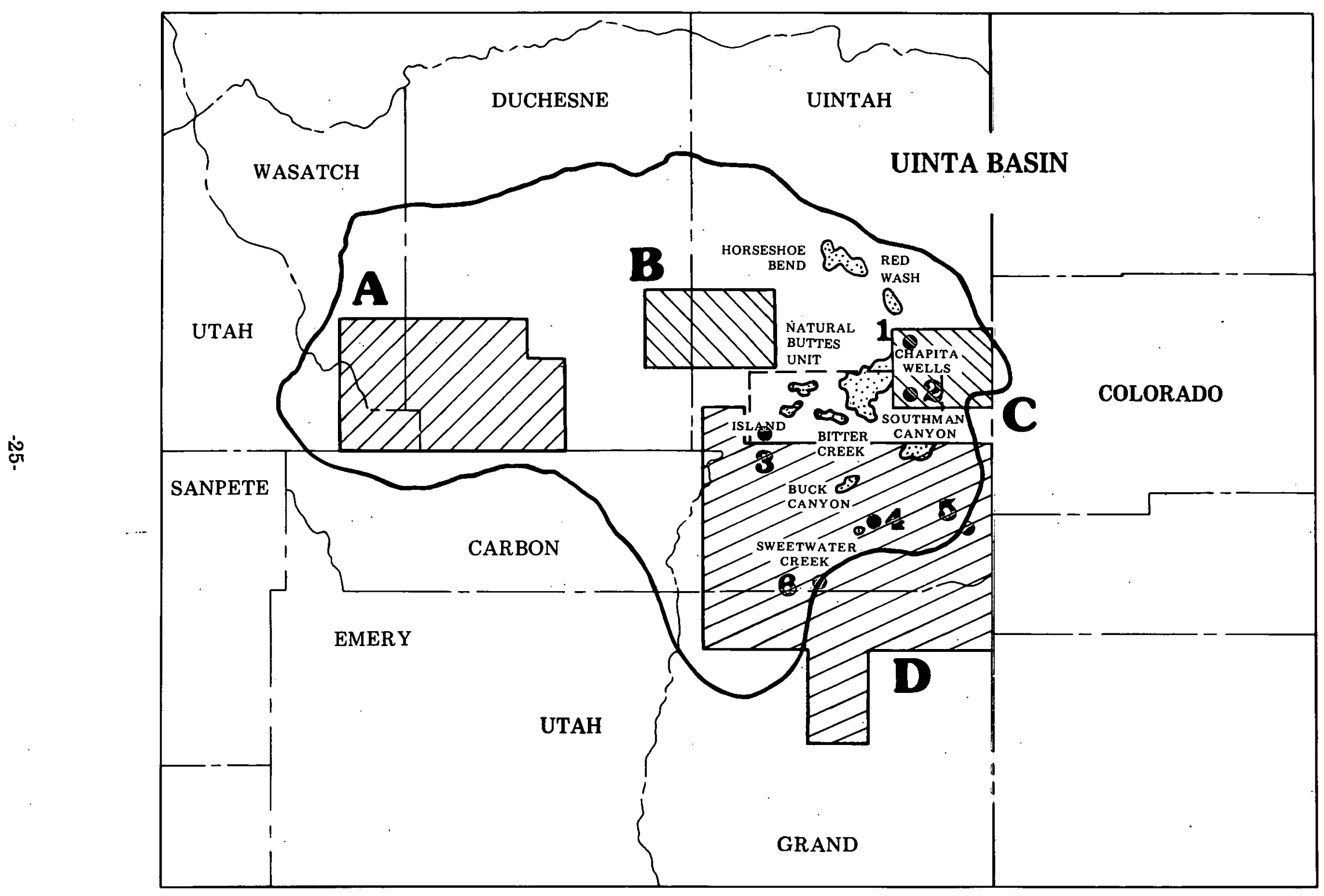

Figure 4-1 USGS Designated Core Areas and Wells of Interest, Uinta Basin (refer to Table 4-1) 
Table 4-1 Summary of Wells Located in USGS Designated Core Areas - Uinta Basin

\begin{tabular}{|c|c|c|c|c|c|c|c|c|c|}
\hline DATE & OPER ATOR & WELL N AME & $\begin{array}{c}\underset{\text { MAP }}{\text { MNDEX }} \\
\text { NO. }\end{array}$ & $\begin{array}{l}\text { LOCATION } \\
\text { Sec/T/R or }\end{array}$ & $\begin{array}{l}\text { HORIzON } \\
\text { (Projected Depth } \\
\text { Producins Interval) }\end{array}$ & $\underset{\text { TD }}{\text { FIN }}$ & $\begin{array}{l}\text { FRACTURE } \\
\text { TREATMENT }\end{array}$ & COMMENTS & $\begin{array}{l}\text { IPF in } \\
\text { MCFD }\end{array}$ \\
\hline $\begin{array}{l}\text { FR: } \\
\text { 9-24-79 }\end{array}$ & $\begin{array}{l}\text { Pacific } \\
\text { Transmission } \\
\text { Supply } \\
\text { Company } \\
\end{array}$ & 23-17 Federal & 1 & $\begin{array}{l}\text { nesw } 17 / 8 S / 23 E \\
\text { WLdcat Field } \\
\text { Uintah Cnty, UT }\end{array}$ & $\begin{array}{l}\text { Mesaverde } \\
\mathbf{8 , 8 0 0}\end{array}$ & & & $\begin{array}{l}\text { W, located in } \\
\text { Core Area C. } \\
\text { Operator has } \\
\text { one additional } \\
\text { Mesaverde test } \\
\text { in this imme- } \\
\text { diate areq. }\end{array}$ & \\
\hline $\begin{array}{l}\text { FR: } \\
\text { 9-19-79 } \\
\quad\end{array}$ & $\begin{array}{l}\text { Belco } \\
\text { Petroleum }\end{array}$ & $\begin{array}{l}\text { 47-30 Chapita } \\
\text { Wells Unit }\end{array}$ & 2 & $\begin{array}{l}\text { nene } 30 / 9 S / 23 E \\
\text { Natural Buttes } \\
\text { Field } \\
\text { Uintah Cnty, UT }\end{array}$ & $\begin{array}{l}\text { Wasatch } \\
\mathbf{7 , 3 8 6}\end{array}$ & & & $\begin{array}{l}\text { D, located in } \\
\text { Core Area C. } \\
\text { Operator has } \\
2 \text { additional } \\
\text { Wasatch tests } \\
\text { in this core area. }\end{array}$ & \\
\hline $\begin{array}{l}\text { FR: } \\
\text { 4-26-78 }\end{array}$ & $\begin{array}{l}\text { Mapco } \\
\text { Incorporated }\end{array}$ & 15-17E RBU & . & $\begin{array}{l}\text { swse } 17 / 10 S / 19 E \\
\text { Island Field } \\
\text { Uintah Cnty, UT }\end{array}$ & $\begin{array}{l}\text { Mesaverde } \\
\mathbf{8 , 4 3 0}\end{array}$ & . & . & $\begin{array}{l}\text { D, Sus, located } \\
\text { in Core Area D. } \\
\text { Operator has } 7 \\
\text { additional Mesa. } \\
\text { verde tests in } \\
\text { this immediate } \\
\text { losale. }\end{array}$ & \\
\hline $\begin{array}{l}\text { FR: } \\
7-20-79\end{array}$ & $\begin{array}{l}\text { Chancellor } \\
\text { \& Ridgeway }\end{array}$ & $\begin{array}{l}4 \text { Rat Hole } \\
\text { Unit }\end{array}$ & 4 & $\begin{array}{l}\text { sesw } 13 / 14 S / 26 E \\
\text { Wildcat Field } \\
\text { Uintah Cnty. UT }\end{array}$ & $\begin{array}{l}\text { Mancos } \\
\mathbf{5 , 6 2 0}\end{array}$ & & & $\begin{array}{l}\text { W. located in } \\
\text { Core Area D. } \\
\text { Operator has } \\
\text { one additional } \\
\text { Mancos test in } \\
\text { this core area. }\end{array}$ & \\
\hline $\begin{array}{l}\text { FH: } \\
\text { 9-24-79 }\end{array}$ & $\begin{array}{l}\text { Coseka } \\
\text { Resources }\end{array}$ & $\begin{array}{l}\text { 3-11-15-21 } \\
\text { Wolf Unit- } \\
\text { Federal }\end{array}$ & 5 & $\begin{array}{l}\text { sesw } 11 / 15 S / 21 E \\
\text { Wildcat Field } \\
\text { Uintah Cnty, UT }\end{array}$ & $\begin{array}{l}\text { Morrison } \\
10,425\end{array}$ & . & & $\begin{array}{l}\text { W. located in } \\
\text { Core Area D. } \\
\text { Operator has } \\
10 \text { additional } \\
\text { tests in this } \\
\text { core area. }\end{array}$ & \\
\hline
\end{tabular}

New gas production totalled 22,621 MCFD for the third quarter, 1979, with all of the production from the Wasatch horizon. New gas production was down 28 percent from the second quarter, 1979. A summary of drilling activities is shown in Table 4-2.

Principal development activity was in the Natural Buttes-Chapita Wells area with Colorado Interstate Gas Exploration and Belco Petroleum being the most active operators.

The largest fracture treatment reported during the third quarter was performed on Wexpro's No. 9 Island Unit well, Sec. 11, T10S, R19E, Uintah County. It was treated with 3,000 gal acid, 76,000 gal gel and 186,000 lb sand and produced 5,247 MCFD.

Summaries of completed wells, and active and newly located wells appear in Tables 4-3 and 4-4, respectively. Figure 4-2 shows the location of these wells. 
Table 4-2 Summary of Drilling Activities, Uinta Basin*

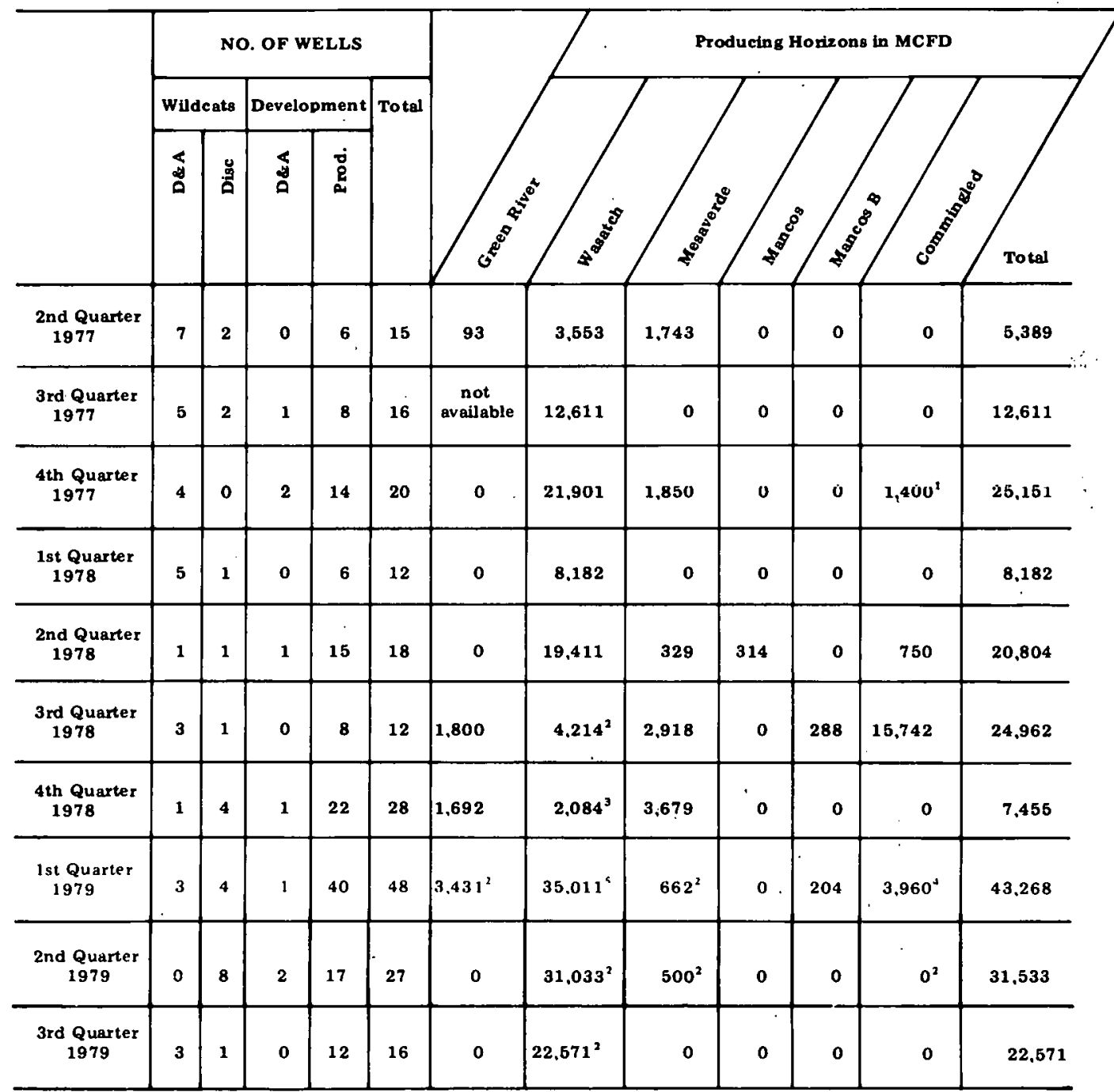

1. Wasatch/Mesaverde

2. IPF not available for one well

3. IPF not avallable for eight wells

4. Mesaverde/Castle gate $-\mathbf{4 1 0}$

Mesaverde $/$ Wasatch - 3,500

5. IPF not available for four wells

*Data compiled from P.I. Rocky Mountain Region Report "Completions" 
Table 4-3 Summary of Wells Completed-Uinta Basin

\begin{tabular}{|c|c|c|c|c|c|c|c|c|c|}
\hline DATE & OPERATOR & WELL NAME & $\begin{array}{c}\text { MAP } \\
\text { INDEX } \\
\text { NO. }\end{array}$ & 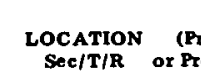 & $\begin{array}{l}\text { HORIzON } \\
\text { rojected Depth } \\
\text { oducing Interval) }\end{array}$ & $\underset{\text { TD }}{\text { FTNAL }}$ & $\begin{array}{l}\text { FRACTURE } \\
\text { TREATMENT }\end{array}$ & COMMENTS & $\begin{array}{l}\text { IPF in } \\
\text { MCFD }\end{array}$ \\
\hline $\begin{array}{l}\text { SP: } \\
5-4-79 \\
\text { CP: } \\
8-13-79\end{array}$ & $\begin{array}{l}\text { Cointinental } \\
\text { Oil }\end{array}$ & $\begin{array}{l}\text { 35-5 Conoco- } \\
\text { Federal }\end{array}$ & 1 & $\begin{array}{l}\text { nwnw 35/8S/21E } \\
\text { Natural Buttes } \\
\text { Field } \\
\text { Uintah Cnty, UT }\end{array}$ & $\begin{array}{l}\text { Wasatch } \\
(5.911-6,483)\end{array}$ & $\begin{array}{l}7.658 \\
\text { PB: } \\
6.700\end{array}$ & $\begin{array}{l}\text { Acidized } \\
\text { Fractured }\end{array}$ & $\begin{array}{l}\text { DG, no cores } \\
\text { or tests. Opera- } \\
\text { tor has } 2 \text { addi- } \\
\text { tional wells in } \\
\text { this area. }\end{array}$ & 1,250 \\
\hline $\begin{array}{l}\text { SP: } \\
11-25-78 \\
\text { CP: } \\
6-6-79\end{array}$ & $\begin{array}{l}\text { Belco } \\
\text { Petroleum } \\
\end{array}$ & $\begin{array}{l}51-31-B \\
\text { Natural } \\
\text { Buttes } \\
\text { Unit }\end{array}$ & 2 & $\begin{array}{l}\text { swse } 31 / 9 S / 21 E \\
\text { Natural Buttes } \\
\text { Field } \\
\text { Uintah Cnty, UT }\end{array}$ & $\begin{array}{l}\text { Wa satch } \\
(5,068-6,838)\end{array}$ & $\begin{array}{l}6,956 \\
\text { PB: } \\
6,873\end{array}$ & & $\begin{array}{l}\text { DG, no cores } \\
\text { or tests. Opera- } \\
\text { tor has } 25 \text { addi- } \\
\text { tional tests in } \\
\text { this a rea. }\end{array}$ & 734 \\
\hline $\begin{array}{l}\text { FR: } \\
4-26 \cdot 78\end{array}$ & $\begin{array}{l}\text { Mapco } \\
\text { Incorporated }\end{array}$ & 5.11D RBU & - & $\begin{array}{l}\text { swnw } 11 / 10 \$ / 18 E \\
\text { Uteland Butte } \\
\text { Field } \\
\text { Uintah Cnty, UT }\end{array}$ & $\begin{array}{l}\text { Mesaverde } \\
8.465\end{array}$ & & & $\begin{array}{l}\mathrm{D}, \text { Sus. Operator } \\
\text { has } 10 \text { additional } \\
\text { tests in this area. }\end{array}$ & \\
\hline $\begin{array}{l}\text { SP: } \\
12-3 \cdot 78 \\
\text { CP: } \\
8-6-79\end{array}$ & Wexpro & 9 Island Unit & 4 & $\begin{array}{l}\text { nwsw } 31 / 10 S / 19 E \\
\text { Island Field } \\
\text { Uintah Cnty, UT }\end{array}$ & $\begin{array}{l}\text { Wasatch } \\
(6,088-6,151)\end{array}$ & $\begin{array}{l}6.800 \\
\text { PB: } \\
6.730\end{array}$ & $\begin{array}{l}\text { Acidized } w / 3,000 \\
\text { gal. Fract } w / 76,000 \\
\text { gal gel, } 186,000 \mathrm{lb} \\
\text { sand }\end{array}$ & $\begin{array}{l}\text { DG, no cores } \\
\text { or tests. Largest } \\
\text { tract reported } \\
\text { this quarter. }\end{array}$ & 5,247 \\
\hline $\begin{array}{l}\text { SP: } \\
\text { 4-14-79 } \\
\text { CP: } \\
6-3-79\end{array}$ & $\begin{array}{l}\text { CIG } \\
\text { Exploration }\end{array}$ & $\begin{array}{l}34-13-10-20 \\
\text { Natural Buttes } \\
\text { Unit }\end{array}$ & $\mathbf{5}$ & $\begin{array}{l}\text { swne } 13 / 10 S / 20 E \\
\text { Natural Buttes } \\
\text { Field } \\
\text { Uintah Cnty, UT }\end{array}$ & $\begin{array}{l}\text { Wasatch } \\
(5,230-6,576)\end{array}$ & $\begin{array}{l}6,700 \\
\text { PB: } \\
6,670\end{array}$ & $\begin{array}{l}\text { Acidized } w / 3,000 \\
\text { gal. Fract } w / 76,342 \\
\text { gal water, } 150,000 \\
\text { lb sand }\end{array}$ & $\begin{array}{l}\text { DG, no cores } \\
\text { or tests. Opera. } \\
\text { tor has } 25 \text { addi- } \\
\text { tional wells in } \\
\text { this area. }\end{array}$ & 3,000 \\
\hline
\end{tabular}

Table 4-4 Summary of Active Wells and New Locations-Uinta Basin

\begin{tabular}{|c|c|c|c|c|c|c|}
\hline DATE & OPERATOR & WELL N AME & $\begin{array}{l}\text { MAP } \\
\text { INDE: } \\
\text { NO. }\end{array}$ & $\begin{array}{l}\text { LOCATION } \\
\text { Sec/T/R }\end{array}$ & $\begin{array}{l}\text { PROJECTED } \\
\text { HORIZON } \\
\text { (Depth in } \mathrm{ft} \text { ) }\end{array}$ & COMMENTS \\
\hline $\begin{array}{l}\text { FR : } \\
\text { 6-29-79 }\end{array}$ & $\begin{array}{l}\text { Belco } \\
\text { Petroleum }\end{array}$ & $\begin{array}{l}\text { 14-34 Stage- } \\
\text { coach }\end{array}$ & 1 & $\begin{array}{l}\text { nenw } 34 / 8 S / 21 E \\
\text { Stagecoach Field } \\
\text { Uintah Cnty, UT }\end{array}$ & $\begin{array}{l}\text { Wasatch } \\
7.525\end{array}$ & $\begin{array}{l}\text { D. Operator has } 3 \text { additional Wasatch } \\
\text { tests in this locale. }\end{array}$ \\
\hline $\begin{array}{l}\text { FR: } \\
9-4-79\end{array}$ & $\begin{array}{l}\text { Pacific } \\
\text { Transmission } \\
\text { Supply } \\
\text { Company }\end{array}$ & $\begin{array}{l}24-22 \\
\text { Federal }\end{array}$ & 2 & $\begin{array}{l}\text { sesw } 22 / 8 S / 22 E \\
\text { Chapita Wells Field } \\
\text { Uintah Cnty, UT }\end{array}$ & $\begin{array}{l}\text { Mesaverde } \\
8,400\end{array}$ & $\begin{array}{l}\text { WO. Operator has } 1 \text { additional Mesa- } \\
\text { verde test in this immediate locale. }\end{array}$ \\
\hline $\begin{array}{l}\text { FR: } \\
9-24-79\end{array}$ & $\begin{array}{l}\text { Mapco } \\
\text { Production }\end{array}$ & $\begin{array}{l}7-25 A \\
\text { Federal }\end{array}$ & 3 & $\begin{array}{l}\text { swne } 25 / 9 S / 18 S \\
\text { Wildcat Field } \\
\text { Uintah Cnty, UT }\end{array}$ & $\begin{array}{l}\text { Wasatch } \\
9,300\end{array}$ & WF. \\
\hline $\begin{array}{l}\text { FR: } \\
\text { 4-26-78 } \\
\text { SP: } \\
7-22-79\end{array}$ & $\begin{array}{l}\text { Mapco } \\
\text { Production }\end{array}$ & $\begin{array}{l}7-25 B \\
\text { Federal } \\
\end{array}$ & 4 & $\begin{array}{l}\text { swne } 25 / 9 S / 19 \mathrm{E} \\
\text { Natural Buttes } \\
\text { Field } \\
\text { Uintah Cnty, UT }\end{array}$ & $\begin{array}{l}\text { Mesaverde } \\
8,730\end{array}$ & $\begin{array}{l}\text { D. TD } 9.232 \text { Rng logs. Well name } \\
\text { chgd from RBU. } \\
\text {. }\end{array}$ \\
\hline $\begin{array}{l}\text { FR: } \\
\text { 5-1-79 } \\
\text { SP: } \\
7-5-79\end{array}$ & $\begin{array}{l}\text { Continental } \\
\text { Oil }\end{array}$ & $\begin{array}{l}\text { 2-6 Ankerpont } \\
\text { et al }\end{array}$ & $\mathbf{5}$ & $\begin{array}{l}\text { nenw } 2 / 9 S / 21 \mathrm{E} \\
\text { Natural Buttes } \\
\text { Field } \\
\text { Uintah Cnty, UT }\end{array}$ & $\begin{array}{l}\text { Wasatch } \\
7,250\end{array}$ & $\begin{array}{l}\text { D. TD } 7.261 \text {, acidized, sdwtrfract, } \\
\text { swbg. }\end{array}$ \\
\hline
\end{tabular}




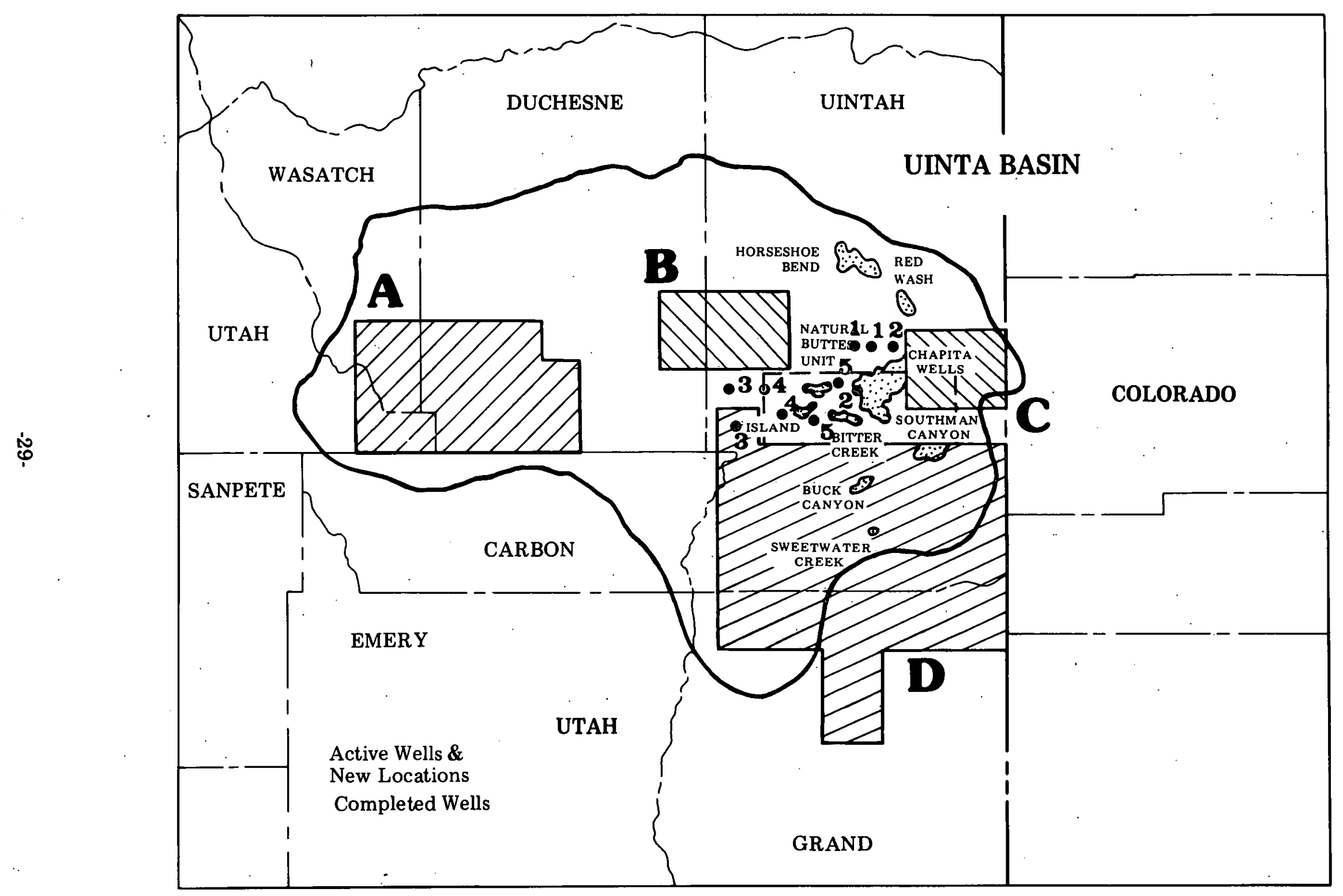

Figure 4-2 Wells Completed and Staked, USGS Designated Core Areas, Uinta Basin (refer to Tables 4-3 and 4-4) 
THIS PAGE

\section{WAS INTENTIONALLY}

LEFT BLANK 


\section{Piceance Basin}

\subsection{CORE PROGRAM}

The Piceance Basin of northwestern Colorado has numerous tight Upper Cretaceous and Tertiary gas sands. Within the Mancos Formation, the Castlegate (Rollins) and the Mancos " $B$ " sands are the principal objectives. The Iles Formation of the Mesaverde Group contains the Corcoran, Cozzette, Sego and Trout Creek sand units which all have gas potential. Within the continental portion of the Mesaverde Group (Williams Fork Formation) and the continental Fort Union and Wasatch Formations, potential is present in both lenticular and blanket sand deposits. Most of these units are productive somewhere within the Piceance Basin. Actively producing gas fields include the Cathedral, Piceance Creek, Trail Canyon, Thunder, Dragon Trail, and Texas Mountain. However, in areas removed from productive fields, or in horizons below known producing zones, very little information is available. For example, in the Piceance Creek Field, very few wells have penetrated below the commercial Wasatch and therefore data is limited.

In an effort to better understand areas or horizons with minimal control, the USGS has recommended several key areas from which cores should be obtained. Within the Piceance Basin these areas are (see Figure 5-1):

Area A

East of Rangely Field in Rio Blanco County, T1S to T2N, R98W to R99W.

Area B

Piceance Creek Field area, Rio Blanco County, T2S, R96W and R97W (below the Wasatch 


\section{Area C}

Garfield and Mesa Counties, T5S to T8S, R95W in the north tapering down to R98W to $\mathrm{R} 99 \mathrm{~W}$ on the southern part of the area.

\section{Area D}

Garfield and Mesa Counties, T8S to T10S, R92W to R94W (this area is flanked on the northwest and southeast by the Grand Mesa National Forest).

Operators are contacted to discuss WGSP core program participation whenever a new well is staked in a designated core area and the well is scheduled to penetrate program objectives. During the third quarter of 1979, David M. Munson was contacted, but declined coring a well near Cathedral Bluffs, Rio Blanco County, Colorado. Also in Rio Blanco County, a contract was obtained with Pacific Transmission Supply Company to core and log portions of Mesaverde/ Williams Fork and Mancos " $B$ " in their well No. 22-12 Federal "Barcus Creek", Sec. 12, T1N, R99W. Coring operations began in August with the recovery of $285 \mathrm{ft}$ of Mesaverde core. Seven cores were cut with 98 percent recovery. Gas was not detected from this Mesaverde section. Selected core samples were sealed and sent to Bartlesville Energy Technology Center, Los Alamos Scientific Laboratory and Lawrence Livermore Laboratory for analyses. Coring of the Mancos " $B$ ", scheduled for mid-September, was delayed due to encountering an unexpectedly thick Mesaverde interval. Core depth was originally projected to be about $10,500 \mathrm{ft}$, but was later revised to $11,500 \mathrm{ft}$.

Core Laboratories, Inc. performed various routine and special analyses on core from the Twin Arrow C\&K No. 4-14 well, Rio Blanco County. Special tests included acoustic velocity measurements, capillary pressure tests, formation resistivity factor measurements at various overburden pressures, and formation resistivity index measurements. 


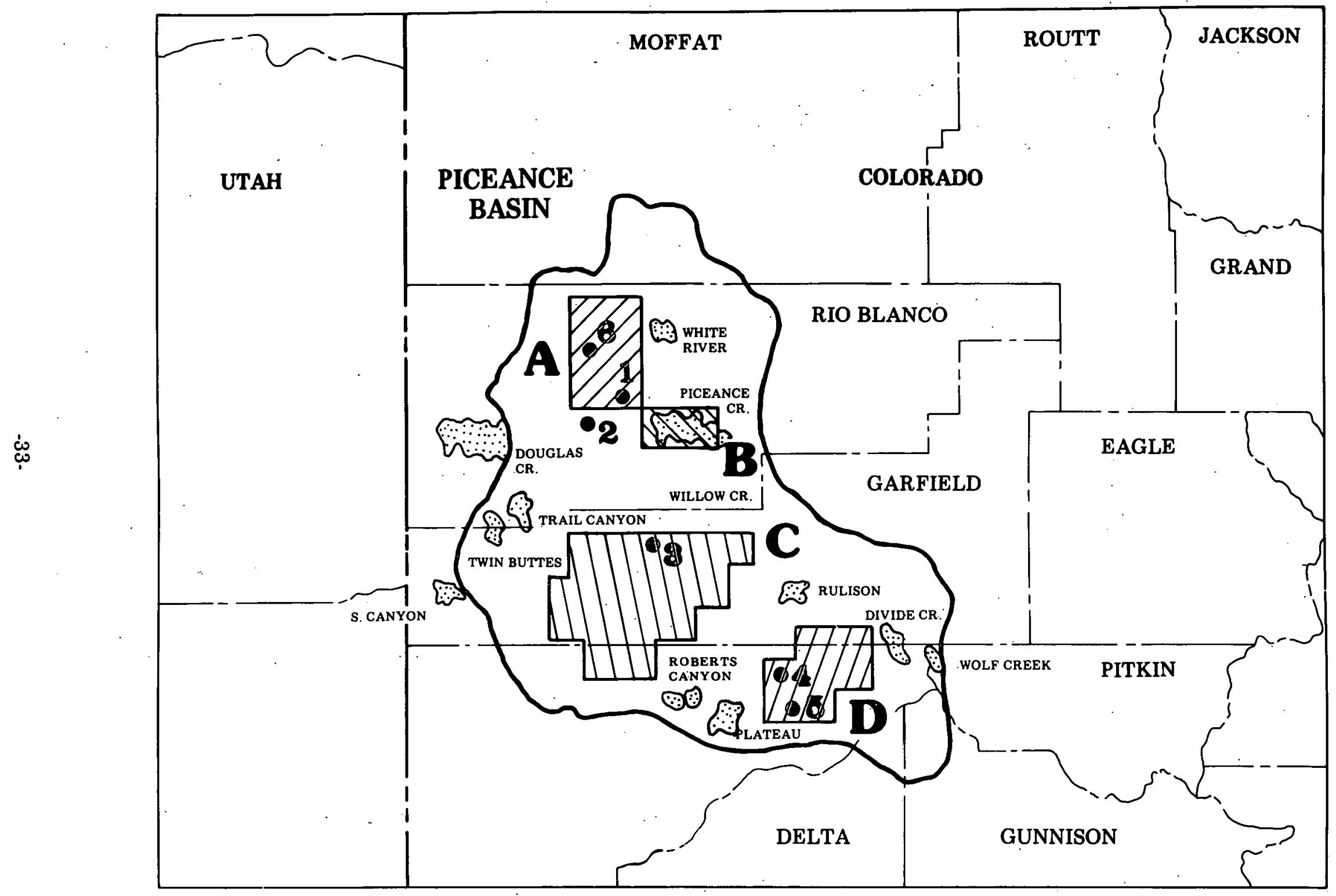

Figure 5-1 USGS Designated Core Areas and Wells of Interest, Piceance Basin (refer to Table 5-1) 
Table 5-1 Summary of Wells Located in USGS Designated Core Areas - Piceance Basin

\begin{tabular}{|c|c|c|c|c|c|c|c|c|c|}
\hline DATE & OPER ATOR & WELL NAME & $\begin{array}{l}\text { MAP } \\
\text { DNEX } \\
\text { NO. }\end{array}$ & $\begin{array}{l}\text { LOCATION } \\
\text { Sec/T/R }\end{array}$ & $\begin{array}{l}\text { HORIzON } \\
\text { rolected Depth } \\
\text { coductng Interval) }\end{array}$ & $\begin{array}{c}\text { FINAL } \\
\text { TD }\end{array}$ & $\begin{array}{l}\text { FRACTURE } \\
\text { TREATMENT }\end{array}$ & COMMENTS & $\begin{array}{l}\text { IPF in } \\
\text { MCFD }\end{array}$ \\
\hline $\begin{array}{l}\text { FR: } \\
\text { 8-21.79 }\end{array}$ & Teton Energy & 23-1 Federal & 1 & $\begin{array}{l}\text { sesw } 23 / 1 \text { S/98W } \\
\text { Widdcat Field } \\
\text { Rlo Blanco Cnty, } \\
\text { Co }\end{array}$ & $\begin{array}{l}\text { Mancos } \\
(12.500)\end{array}$ & & & $\begin{array}{l}\text { WF. located in } \\
\text { Core Area A. } \\
\text { Operator has } 3 \\
\text { additional wells } \\
\text { in the core area. }\end{array}$ & \\
\hline $\begin{array}{l}\text { FR: } \\
\text { 7-26-79 }\end{array}$ & David M. Munson & 11-2-99 Federal & 2 & $\begin{array}{l}\text { swnw } 11 / 2 \text { S/99w } \\
\text { Wild cat Fleld } \\
\text { Rio Blanco Cnty. } \\
\text { Co }\end{array}$ & $\begin{array}{l}\text { Mancos } \\
(11,000)\end{array}$ & & & $\begin{array}{l}\text { WF, located in } \\
\text { Core Area A. } \\
\text { Operator has } 1 \\
\text { additional well } \\
\text { in core area. }\end{array}$ & \\
\hline $\begin{array}{l}\text { FR: } \\
\text { 9-11.79 }\end{array}$ & Adolph Coors & 1-36 USA-DF & 3 & $\begin{array}{l}\text { swne } 36 / 7 \text { S/99W } \\
\text { Wild cat Field } \\
\text { Garfield Cnty, co }\end{array}$ & $\begin{array}{l}\text { Mancos } \\
(4,000)\end{array}$ & & & $\begin{array}{l}\text { WF, located in } \\
\text { Core Area C. } \\
\text { Operator has } 1 \\
\text { additlonal well } \\
\text { in immediate } \\
\text { area. }\end{array}$ & \\
\hline $\begin{array}{l}\text { SP: } \\
\text { 6-24-79 } \\
\text { CP: } \\
6-19-79\end{array}$ & $\begin{array}{l}\text { Chandlez \& } \\
\text { Associates }\end{array}$ & 16-29 Pruet! & 4 & $\begin{array}{l}\text { swse } 29 / 9 \mathrm{~S} / 95 \mathrm{~W} \\
\text { Wildcat Field } \\
\text { Mesa Cnty, co }\end{array}$ & $\begin{array}{l}\text { Corcoran } \\
(4,995)\end{array}$ & 4,996 & & $\begin{array}{l}\text { WF, D\& A. } \\
\text { Located in } \\
\text { Core Area D. } \\
\text { Operatox has } \\
6 \text { additional } \\
\text { wells in locale. }\end{array}$ & \\
\hline $\begin{array}{l}\text { FR: } \\
\text { 7-26-79 }\end{array}$ & $\begin{array}{l}\text { Exxon } \\
\text { Corporation }\end{array}$ & 1 Federal & 5 & $\begin{array}{l}\text { sese } 33 / 10 \mathrm{OS} / 94 \mathrm{~W} \\
\text { Wudcat Field } \\
\text { Mese Cnty. Co }\end{array}$ & $\begin{array}{l}\text { Corcoran } \\
(8,600)\end{array}$ & & ' & $\begin{array}{l}\text { WF, located in } \\
\text { Core Area D. } \\
\text { Operator has } \\
2 \text { additional } \\
\text { wells in locale. }\end{array}$ & \\
\hline $\begin{array}{l}\text { FR: } \\
6-1-79 \\
\text { SP: } \\
7-10-79\end{array}$ & $\begin{array}{l}\text { Pacific } \\
\text { Transmisston } \\
\text { Supply } \\
\text { Compeny }\end{array}$ & 22-12 Federal & 6 & $\begin{array}{l}\text { senw } 12 / 1 \mathrm{~N} / 99 \mathrm{~W} \\
\text { Wildcat Field } \\
\text { Rio Blanco Cnty. } \\
\text { Co }\end{array}$ & $\begin{array}{l}\text { Dakota } \\
(14,500)\end{array}$ & & & $\begin{array}{l}\text { WF, Drlg 10,142. } \\
\text { Located in Core } \\
\text { Area A. (See } \\
\text { text for more } \\
\text { in formation). }\end{array}$ & \\
\hline
\end{tabular}

A summary of core area wells is listed in Table 5-1 and the locations are shown in Figure 5-1.

\subsection{DRILLING ACTIVITIES}

Third quarter activity was up substantially in the Piceance Basin. Thirty-one wells were reported completed in the Upper Cretaceous and lower Tertiary horizons. Twenty-seven were development wells, with 25 producing and 2 D\&A. Of 4 wildcat wells, 1 was a producer and 3 were $\mathbf{D} \& A$.

New production for the quarter totalled 22,866 MCFD. A substantial increase in Mesaverde production accounted for the 348 percent increase over the previous quarter. The Mesaverde group provided 39 percent of total production, the Mancos " $\mathrm{B}$ " contributed 30 percent, commingled horizons (including Cozzette, Corcóran, Rollins, Mancos B, Mesaverde and Dakota) produced 20 percent and the undifferentiated Mancos provided 5 percent of total production. The quarterly tabulation of production is summarized in Table 5-2. 
Table 5-2 Summary of Drilling Activities, Piceance Basin *

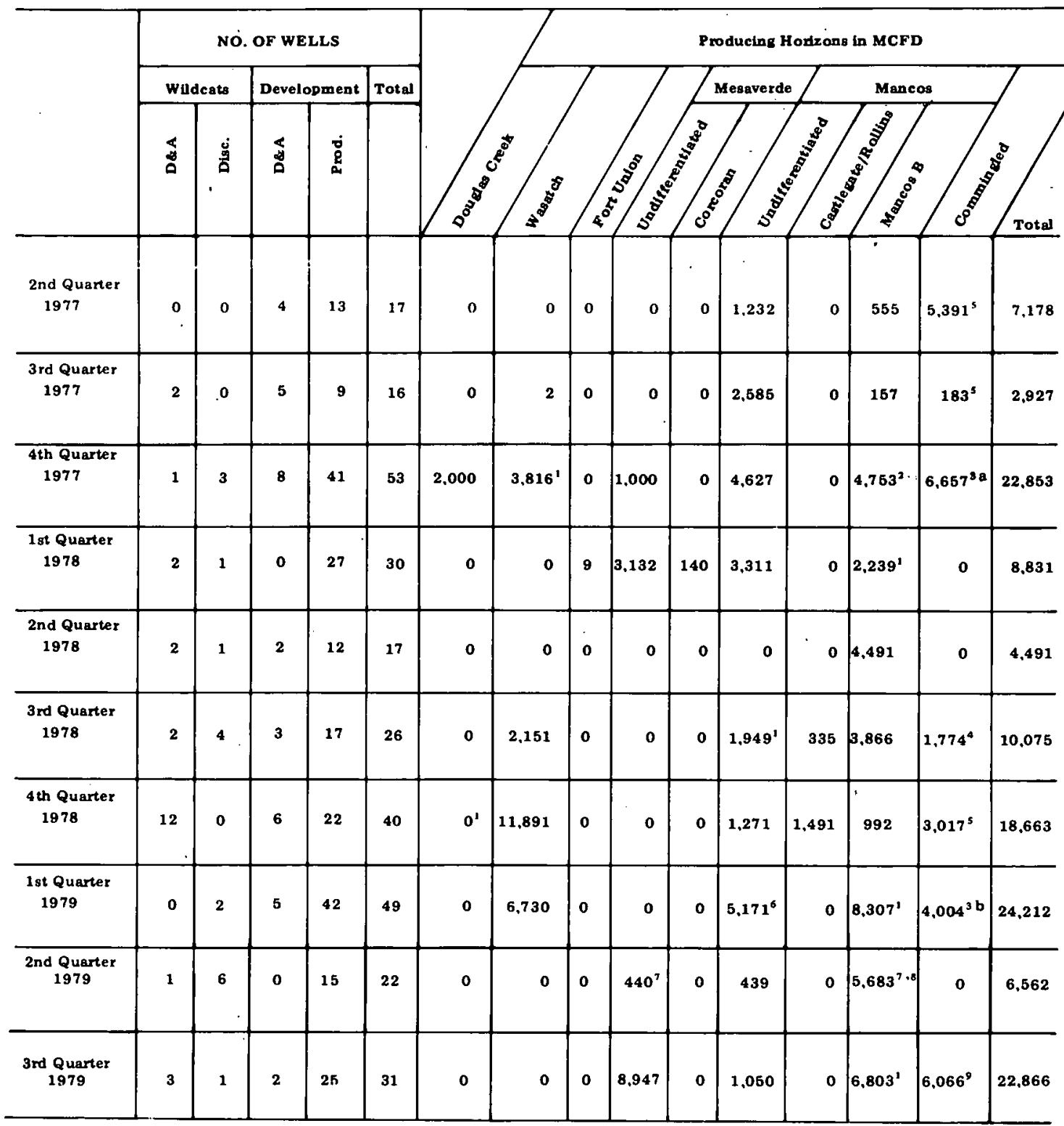

\footnotetext{
1. IPF not available for one well.

2. IPF not available for two wells.

3a Rollins/Cozzette/Coreoran - 4,422

Cozzette/Corcoran - 2,235

3b. Rollins/Corcoran/Cozzette $\cdot \mathbf{1 , 2 8 4}$ Corcoran/Cozzette $-\mathbf{2 . 7 2 0}$

4. Cameo/Cozzette/Corcoran-460 Morapos/Mancos - 481

Rollins/Cozzette/Corcoran - $\mathbf{8 3 3}$
}

5. Rollins/Cozzette/Corcoran - 183

6. IPF not available for five wells.

7. IPF not available for three wells.

8. Natural Production 2,675

9. Cozzette/Corcoran $\mathbf{2} \mathbf{2 , 5 3 7}$

Rollins/Cozzet te/Corcoran - 1,087

Mancos B/Dakota - $\mathbf{2 . 4 2 8}$

Mancos/Mesaverde - i4

"Data compiled from P.1. Rocky Mountain Region Report "Completions," 
The Mesaverde production was mainly from the Plateau and DeBeque Fields in Mesa County. In Rio Blanco County, the Douglas Creek Arch, including the Cathedral Field, was an active area, with the main objective being the Mancos B (Emery).

The largest fracture treatment reported for the quarter was performed on the Fuel Resources Development J-36-2-101-S Federal well, Sec. 36, T2S, R101W, Cathedral Field, Rio Blanco County. The well produced 1,729 MCFD from the Emery after being fractured with $100,800 \cdot \mathrm{gal}$ fluid, $400,000 \mathrm{lb}$ sand and $1,080,000 \mathrm{scf}$ nitrogen.

Summaries of completed wells and active and newly located wells are in Tables 5-3 and 5-4, respectively. Figure 5-3 shows the location of these wells.

Table 5-3 Summary of Wells Completed-Piceance Basin

\begin{tabular}{|c|c|c|c|c|c|c|c|c|c|}
\hline DATE & OPER ATOR & WELL NAME & $\begin{array}{l}\text { MAP. } \\
\text { INDEX } \\
\text { NO. }\end{array}$ & $\underset{\text { Sec/T/R }}{\text { LOCATION }}$ or $\mathrm{P}$ & $\begin{array}{l}\text { HORIZON } \\
\text { rojected Depth } \\
\text { roducing Interval) }\end{array}$ & $\underset{\text { TD }}{\text { FINAL }}$ & $\begin{array}{l}\text { FRACTURE } \\
\text { TREATMENT }\end{array}$ & COMMENTS & $\begin{array}{l}\text { IPF in } \\
\text { MCFD }\end{array}$ \\
\hline $\begin{array}{l}\text { SP: } \\
12-21-78 \\
\text { CP: } \\
7-27-79\end{array}$ & $\begin{array}{l}\text { Fuel Resources } \\
\text { Development }\end{array}$ & $\begin{array}{l}\text { J-36-2-101-S } \\
\text { Federal }\end{array}$ & 1 & $\begin{array}{l}\text { nwse } 36 / 2 S / 101 w \\
\text { Cathedral Field } \\
\text { Rio Blanco Cnty, } \\
\text { Co }\end{array}$ & $\begin{array}{l}\text { Emery } \\
(2,632-2,870)\end{array}$ & $\begin{array}{l}\text { 3,042 } \\
\text { PB: } \\
2,979\end{array}$ & $\begin{array}{l}100,800 \mathrm{gal} \text { water } \\
400,000 \mathrm{lb} \text { sand } \\
1,080,000 \mathrm{sct} \\
\text { nitrogen }\end{array}$ & $\begin{array}{l}\text { DG, no cores } \\
\text { or tests. Largest } \\
\text { fract reported in } \\
\text { quarter. Opera- } \\
\text { tor has } 11 \text { addi- } \\
\text { tional wells in } \\
\text { grea. }\end{array}$ & 1,729 \\
\hline $\begin{array}{l}\text { SP: } \\
\text { 6-24-79 } \\
\text { CP: } \\
\text { 8-13-79 }\end{array}$ & $\begin{array}{l}\text { Chandler \& } \\
\text { Associates }\end{array}$ & 15-33 Stites & 2 & $\begin{array}{l}\text { swse 33/9S/95W } \\
\text { Plateau Field } \\
\text { Mesa Cnty, CO }\end{array}$ & $\begin{array}{l}\text { Cozzette } \\
(4,644-4,673) \\
\text { Corcoran } \\
(4,779-4,863)\end{array}$ & $\begin{array}{c}6,100 \\
.\end{array}$ & $\begin{array}{l}\text { Acidized } w / 750 \mathrm{gal} \text {. } \\
\text { fract } w / 41,625 \mathrm{gal} \\
\text { water }\end{array}$ & $\begin{array}{l}\text { WOE, comming- } \\
\text { led.production. } \\
\text { Cozzette Exten- } \\
\text { sion-Plateau Field } \\
\text { Operator has } 3 \\
\text { additional wells } \\
\text { in locale. }\end{array}$ & 490 \\
\hline $\begin{array}{l}\text { SP: } \\
4-20-79 \\
\text { CP: } \\
7-11-79\end{array}$ & $\begin{array}{l}\text { Chandler \& } \\
\text { Associates }\end{array}$ & 2-4 Gunderson & 3 & $\begin{array}{l}\text { nwne } 4 / 10 S / 96 w \\
\text { Plateau Feld } \\
\text { Mesa Cnty, Co }\end{array}$ & $\begin{array}{l}\text { Corcoran } \\
(4,365)\end{array}$ & 4,500 & & $\begin{array}{l}\text { WO, D\& A. No } \\
\text { cores or tests. } \\
\text { Operator has one } \\
\text { additional well } \\
\text { in locale. }\end{array}$ & \\
\hline $\begin{array}{l}\text { SP: } \\
12-7-77 \\
\text { CP: } \\
10-17-78\end{array}$ & Adolph Coors & 1-32 Acco-Wood & $\mathbf{3}$ & $\begin{array}{l}\text { sese } 32 / 10 \mathrm{O} / 96 \mathrm{~W} \\
\text { Plateau Fleld } \\
\text { Mesa Cnty, Co }\end{array}$ & $\begin{array}{l}\text { Cozzette } \\
(3,402-3,440) \\
\text { Corcoran } \\
(3,635-3,588)\end{array}$ & 4.242 & $\begin{array}{l}289,125 \mathrm{gal} \text { water } \\
522,000 \mathrm{lb} \text { sand }\end{array}$ & $\begin{array}{l}\text { DG, no cores or } \\
\text { tests. Operator } \\
\text { has } 5 \text { additional } \\
\text { wells in locale. }\end{array}$ & 474 \\
\hline $\begin{array}{l}\text { SP: } \\
9-18-78 \\
\text { CP: } \\
4-23-79\end{array}$ & $\begin{array}{l}\text { Fuel Resources } \\
\text { Development }\end{array}$ & 6M Federal & 4 & $\begin{array}{l}\text { nesw } 20 / 2 \mathrm{~N} / 96 \mathrm{~W} \\
\text { White River Field } \\
\text { Rio Blanco Cnty, } \\
\text { CO }\end{array}$ & $\begin{array}{l}\text { Mesaverde } \\
(6,170-6,862)\end{array}$ & $\begin{array}{l}7.548 \\
\text { PB: } \\
7.040\end{array}$ & $\begin{array}{l}\text { Acidized } w / 1,596 \\
\text { gal, fract } w / 6,720 \\
\text { gal water, } 25,000 \\
\text { lb sand }\end{array}$ & $\begin{array}{l}\text { DG, no cores } \\
\text { or tests. }\end{array}$ & 3,691 \\
\hline $\begin{array}{l}\text { CP: } \\
7-4-79\end{array}$ & Teton Energy & $\begin{array}{l}\text { 25-2 Indian } \\
\text { Valley-Federal }\end{array}$ & 5 & $\begin{array}{l}\text { senw } 26 / 3 N / 97 w \\
\text { Wildcat Field } \\
\text { Rio Blanco Cnty, } \\
\text { Co }\end{array}$ & $\begin{array}{l}\text { Morapos } \\
(8.000)\end{array}$ & 8,000 & & WF, D\&A. & \\
\hline
\end{tabular}


Table 5-4 Summary of Active Wells and New Locations-Piceance Basin

\begin{tabular}{|c|c|c|c|c|c|c|}
\hline DATE & OPERATOR & WELL NAME & $\begin{array}{r}\text { MAP } \\
\text { LNDEX } \\
\text { NO. }\end{array}$ & $\begin{array}{l}\text { LOCATION } \\
\text { Sec/T/R }\end{array}$ & $\begin{array}{l}\text { PROJECTED } \\
\text { HORIZON } \\
\text { (Depth in } \mathrm{ft} \text { ) }\end{array}$ & COMMENTS \\
\hline $\begin{array}{l}\text { FR: } \\
\text { 9-13-79 }\end{array}$ & Adolph Coors & $\begin{array}{l}\text { 1-20 USA- } \\
\text { Philadelphia } \\
\text { Creek }\end{array}$ & $\begin{array}{ll}1 & s \\
& \\
& R\end{array}$ & $\begin{array}{l}\text { swnw } 20 / 1 \mathrm{~S} / 100 \mathrm{~W} \\
\text { Wildcat Field } \\
\text { Rio Blanco Cnty. co }\end{array}$ & $\begin{array}{l}\text { Mancos } \\
5,500\end{array}$ & WF. \\
\hline $\begin{array}{l}\text { FR: } \\
\text { 5-18-79 } \\
\text { SP: } \\
7-23-79\end{array}$ & $\begin{array}{l}\text { Fuel Resources } \\
\text { Development }\end{array}$ & $\begin{array}{l}\text { P 23-3-101S } \\
\text { Federal }\end{array}$ & $\begin{array}{ll}2 & \mathrm{~s} \\
& \mathrm{C} \\
\mathrm{R} & -1\end{array}$ & $\begin{array}{l}\text { sese } 23 / 3 \mathrm{~S} / 101 \mathrm{~W} \\
\text { Cathedral Field } \\
\text { Rio Blanco Cnty, co }\end{array}$ & $\begin{array}{l}\text { Emery } \\
3,892\end{array}$ & $\begin{array}{l}\text { D. TD } 3,815 \text {, ran prod csg, fract, } F \\
\text { tstg. Operator has } 12 \text { additional } \\
\text { wells in immediate locale. }\end{array}$ \\
\hline $\begin{array}{l}\text { FR: } \\
\text { 7-9-79 }\end{array}$ & $\begin{array}{l}\text { Northwest } \\
\text { Exploration }\end{array}$ & $\begin{array}{l}7 \text { Texas } \\
\text { Creek }\end{array}$ & $\begin{array}{ll}3 & s \\
\text { u } \\
\mathrm{R}\end{array}$ & $\begin{array}{l}\text { swne } 15 / 3 \mathrm{~S} / 103 \mathrm{~W} \\
\text { Wildcat Field } \\
\text { Rio Blanco Cnty, co }\end{array}$ & $\begin{array}{l}3,500 \\
\text { test }\end{array}$ & $\begin{array}{l}\text { WF. Operator has } 6 \text { additional tests } \\
\text { in immediate locale. }\end{array}$ \\
\hline $\begin{array}{l}\text { FR: } \\
10-26-78 \\
\text { SP: } \\
2-24-79\end{array}$ & $\begin{array}{l}\text { Northwest } \\
\text { Exploration }\end{array}$ & 2 Clough & $\begin{array}{l}4 . s \\
\text { R } \\
\mathrm{G}\end{array}$ & $\begin{array}{l}\text { sesw } 21 / 6 S / 94 \mathrm{~W} \\
\text { Rulison Field } \\
\text { Garfield Cnty, CO }\end{array}$ & $\begin{array}{l}\text { Mancos } \\
8,700\end{array}$ & $\begin{array}{l}\text { WOE, TD } 8,600 \text {, ran prod csg, perf. } \\
\text { acidized. Mesaverde prod zone. } \\
\text { holding for data. }\end{array}$ \\
\hline $\begin{array}{l}\text { FR: } \\
\text { 7-11-79 }\end{array}$ & Norris Oil & 9-1 Federal & $\begin{array}{ll}5 \\
n \\
R \\
M\end{array}$ & $\begin{array}{l}\text { nenw } 9 / 10 S / 97 \mathrm{~W} \\
\text { Roberts Canyon } \\
\text { Mesa Cnty, CO }\end{array}$ & $\begin{array}{l}\text { Corcoran } \\
2,890\end{array}$ & $\begin{array}{l}\text { WS. Operator has } 3 \text { additional } \\
\text { Corcoran tests in immediate area. }\end{array}$ \\
\hline
\end{tabular}




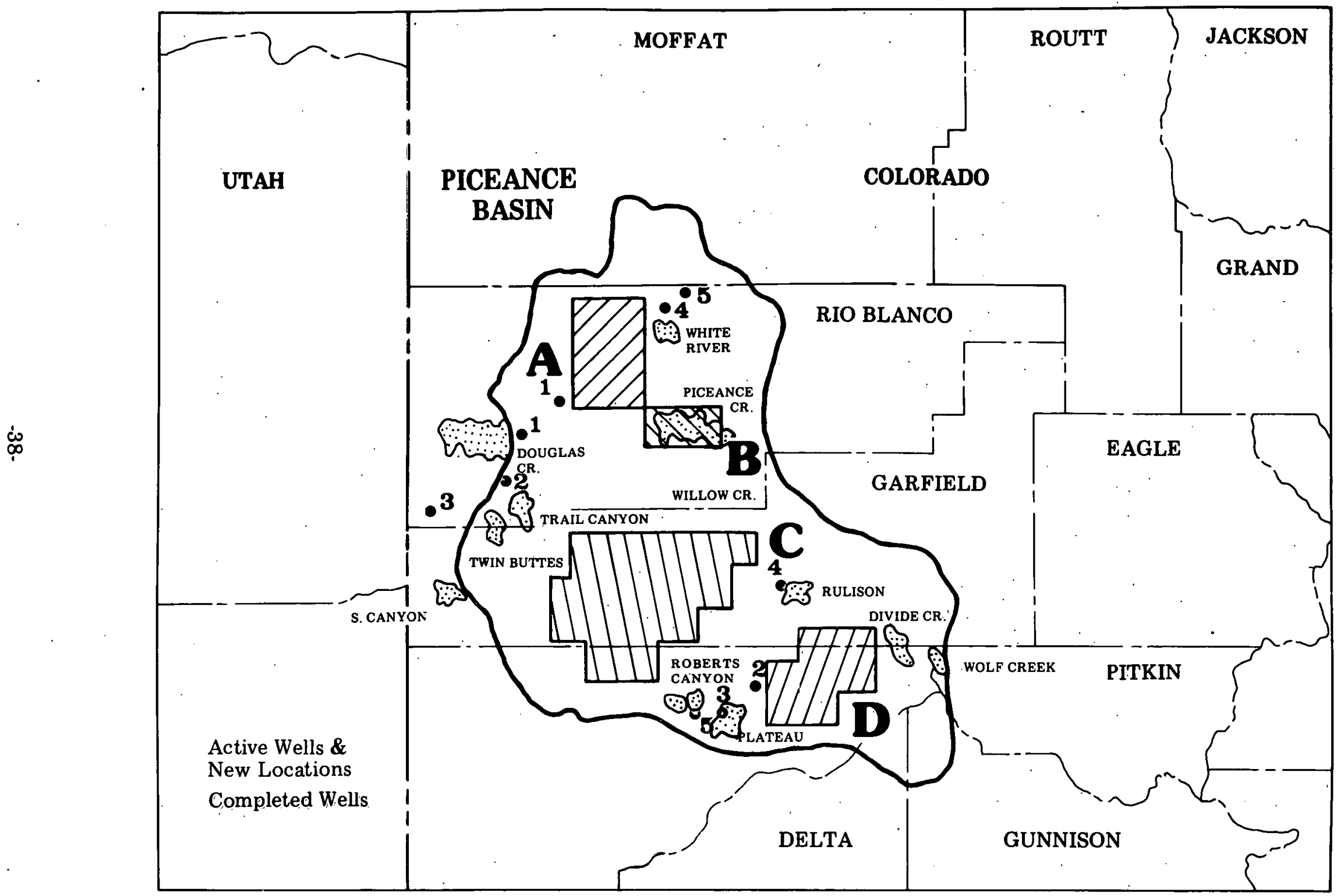

Figure 5-2 Wells Completed and Staked, USGS Designated Core Areas, Piceance Basin (refer to Tables 5-3 and 5-4) 